Discussion Paper No. 621

SIGNAL QUALITIES, ORDER OF DECISIONS, AND INFORMATIONAL CASCADES:

EXPERIMEMTAL EVIDENCES

Shunichiro Sasaki

October 2004

The Institute of Social and Economic Research Osaka University

6-1 Mihogaoka, Ibaraki, Osaka 567-0047, Japan 


\title{
Signal Qualities, Order of Decisions, and Informational Cascades: Experimental Evidences ${ }^{1}$
}

\author{
Shunichiro Sasaki ${ }^{2}$ \\ Institute of Social and Economic Research \\ Osaka University
}

\footnotetext{
${ }^{1}$ The author acknowledges financial supports from Keio University and the 21st century COE program of Osaka University.

${ }^{2}$ Address: Institute of Social and Economic Research, Osaka University, 61 Mihogaoka, Ibaraki, Osaka 567-0047, Japan, Telephone: +81-6-6877-5111 (ex. 9179), Facsimile: +81-6-6878-2766, Email: ssasaki@iser.osaka-u.ac.jp
} 


\begin{abstract}
This paper reports the results of informational cascades experiments where two different decision-making systems, anti-seniority and seniority are investigated. By implementing heterogeneous signal qualities associated with the fixed order of decisions I compare the property of each system and examine heuristics human subjects use. Major findings are the following: (1) complete cascades occur more frequently in seniority than in anti-seniority, (2) seniority is more efficient than anti-seniority, but it increases the risk for creating negative cascades, (3) for both systems, rational complete cascades occur less frequently than those which the Bayesian theory predicts, (4) subjects in seniority put equal weight on private signals and on predecessors' predictions whereas those who in anti-seniority put more weight on private signals than on predecessors' predictions, (5) by analyzing deviations from Bayesian posteriors, both overconfidence and underconfidence are identified, and (6) the anchoring effect is verified in deviations by overconfidence, but is not verified in deviations by underconfidence.
\end{abstract}

Keywords: Informational cascades, experiment, signal quality, overconfidence, underconfidence

JEL classification: C91, C92, D81. 


\section{Introduction}

Suppose a situation where individuals have informative but noisy private signals for an underlying state and they decide in sequence appropriate actions for that state. If individuals' actions are publicly observable, successors can infer their predecessors' private signals. These publicly inferred signals are aggregated as the decision-making process goes on. When an individual's private signal does not correspond to the aggregated publicly inferred signals the rational individual may ignore it and follow the actions the majority of predecessors have chosen. Once an identical pattern of actions is established, private signals are no longer informative, and informational cascades occur. Bikhchandani, S., D. Hirshleifer, and I. Welch (1992) (hereafter BHW) and Bannerjee, A. V. (1992) formulated such a situation and showed that informational cascades are caused by rational Bayesian update.

So far, many experiments have been conducted to test BHW's theory of informational cascades and its empirical applicability. In the pioneering experiment by Anderson, L. R. and C. A. Holt (1997), they directly tested BHW's model of informational cascades by implementing binary-signal and binary-action framework. Over 56 periods where informational cascades were possible in the symmetrically identical signal quality treatment, positive cascades occurred in 28 periods and negative cascades occurred in 13 periods ${ }^{1}$ while any cascades did not develop in 15 periods. Nöth, M. and M. Weber (1999) extended Anderson and Holt 's experiment by introducing two different qualities of signals and found that although informational cascades occurred the frequency of occurrences is lower than the Bayesian theory suggests because human subjects employed a heuristic, which puts too much weight on their private signals. This tendency of overconfidence on private signals is observed in hypothetical experiment (Huck, S. and J. Oechssler (2000) ) and is invariant even when acquisition of signals is costly (Kraemer, C., M. Nöth, and M. Weber (2000)). Kraemer, T. and M. Nöth (2000) tried to identify the "anchoring and adjustment" effect in the Bayesian updating process. By having subjects submit their probability judgements before making predictions, they concluded that subjects' decisions are anchored by their private signals and adjusted by predecessors' predictions. Oberhammer, C. and A. Stiehler (2001) introduced binary-signal and continuous-action model while Çelen, B. and S. Kariv (2004) implemented coutinuous-signal and binary-action framework in order to find out subjects' heuristics.

What we have known from these experiments is that informational cas-

\footnotetext{
${ }^{1}$ In the terminology used in this paper, this includes both complete and partial cascades. For detail, see section 4.1.
} 
cades certainly occur as BHW suggests, but actual occurrences are consistently fewer than the Bayesian theory predicts. This can be attributed to heuristics human subjects use in the process of probabilistic inference. Especially, tendency to putting more weight on private signals than on predecessors' predictions is often observed in many experiments.

In this paper I tested the occurrence of informational cascades by introducing heterogenous qualities of private signals associated with the fixed orders in making predictions. In a series of experiments I examined two systems, anti-seniority and seniority and compared each efficiency, potentiality for cascades occurrence, and ability of extracting private signals. Major findings are the following. Complete cascades occur more frequently in seniority than in anti-seniority. Seniority is more efficient than anti-seniority, but it increases the risk for creating negative cascades. For both treatments, rational complete cascades occur less frequently than those which the Bayesian theory predicts. Private signals can be extracted more effectively in anti-seniority than in seniority. In the process of decision-making, subjects in seniority put equal weight on private signals and on predecessors' predictions whereas subjects in anti-seniority put more weight on private signals than on predecessors' predictions. For heuristics human subjects use, both overconfidence and underconfidence are identified. In addition, the anchoring effect of private signals is verified in deviations by overconfidence, but is not verified in deviations by underconfidence.

The remaining paper is organized as follows. Section 2 introduces analytical framework and presents theoretical predictions, section 3 describes experimental procedure, section 4 reports the result and discusses its implications, and section 5 concludes.

\section{Analytical Framework}

There are two states of the world $\omega \in\{A, B\}$. Each state realizes equally likely, that is $\operatorname{Pr}(A)=p=1 / 2$ and $\operatorname{Pr}(B)=1-p=1 / 2$. Each individual does not know which state would have realized. However, he or she receives an informative but noisy private signal and can infer the realized state by observing it. Let $\sigma_{\omega}$ be the private signal which indicates that realized state is $\omega$. Then, the assumption of noisy information leads to $1 / 2<\operatorname{Pr}\left(A \mid \sigma_{A}\right)<1$ and $1 / 2<\operatorname{Pr}\left(B \mid \sigma_{B}\right)<1$. Specifically I use heterogenous discrete signals with six different qualities from .55 to .8 incremented by .05 . That is, $\operatorname{Pr}\left(A \mid \sigma_{A}\right)$ or $\operatorname{Pr}\left(B \mid \sigma_{B}\right)$ is drawn from the set $\{.55, .6, .65, .7, .75, .8\}$ without replacement. Six subjects participate in each experimental session and each of them makes a prediction which state would have realized one by one in sequence. The 
quality of signal is exogenously determined by the position where a subject is assigned to. Let $i \in\{1,2,3,4,5,6\}$ be the position, $\sigma_{\omega}^{i}$ be the signal the subject in position $i$ receives, and $\pi_{\omega}^{i}$ be the prediction he or she makes.

In addition to private signals, each subject can observe all predictions made by his or her predecessors. For example, the subject in position 2 can observe the prediction made by the subject in position 1 , the subject in position 6 (last position) can observe the five predictions made by the subjects in positions from 1 to 5 , and so on. Thus, the subjects assigned to later than position 2 make predictions by referring to both private signals and their predecessors' predictions whereas the subject in position 1 can observe only his or her private signal.

In experimental sessions, two treatments anti-seniority ${ }^{2}$ and seniority are conducted. The difference of each treatment is in the combinations of signal qualities and orders of making predictions, which are summarized in table 1.

- Anti-seniority

In anti-seniority treatment, six subjects make predictions in ascending order of the signal qualities. That is, the subject who has the least precise signal makes the prediction in position $1\left(\operatorname{Pr}\left(A \mid \sigma_{A}^{1}\right)=\operatorname{Pr}\left(B \mid \sigma_{B}^{1}\right)=.55\right)$, the subject who has the second least precise signal makes the prediction in position $2\left(\operatorname{Pr}\left(A \mid \sigma_{A}^{2}\right)=\operatorname{Pr}\left(B \mid \sigma_{B}^{2}\right)=.6\right)$, and in this manner, the subject who has the most precise signal makes the prediction in position 6 $\left(\operatorname{Pr}\left(A \mid \sigma_{A}^{6}\right)=\operatorname{Pr}\left(B \mid \sigma_{B}^{6}\right)=.8\right)$.

- Seniority

In seniority treatment, six subjects make predictions in descending order of the signal qualities. That is, the subject who has the most precise signal makes the prediction in position $1\left(\operatorname{Pr}\left(A \mid \sigma_{A}^{1}\right)=\operatorname{Pr}\left(B \mid \sigma_{B}^{1}\right)=.8\right)$, the subjects who has the second most precise signal makes the prediction in position $2\left(\operatorname{Pr}\left(A \mid \sigma_{A}^{2}\right)=\operatorname{Pr}\left(B \mid \sigma_{B}^{2}\right)=.75\right)$, and in this manner, the subject who has the least precise signal makes the prediction in position 6 $\left(\operatorname{Pr}\left(A \mid \sigma_{A}^{6}\right)=\operatorname{Pr}\left(B \mid \sigma_{B}^{6}\right)=.55\right)$.

In experimental sessions, the combinations of signal qualities and order of decisions are common knowledge among all subjects. Thus, rational subjects can calculate posterior probability by using Bayes' rule. If they do so, these two different treatments would create different behavioral patterns in the aggregate as the following.

\footnotetext{
${ }^{2}$ The term anti-seniority is used in Ottaviani, M. and P. N. Sørensen (2001).
} 
In anti-seniority, imbalances between Bayesian posterior probability and private signal occur only at 44 out of 252 decision nodes ${ }^{3}$. Complete informational cascades ${ }^{4}$ can occur if subjects in the first three consecutive positions make the same predictions. For example, the posterior probability that state A would have realized given that the subjects in the first three consecutive positions have predicted $\mathrm{A}$ and the subject in position 4 observes $\sigma_{B}^{4}$ is $\operatorname{Pr}\left(A \mid \pi_{A}^{1}, \pi_{A}^{2}, \pi_{A}^{3}, \sigma_{B}^{.4}\right)=\frac{\frac{1}{2} \times .55 \times .6 \times .65 \times .3}{\frac{1}{2} \times[(.55 \times .6 \times .65 \times .3)+(.45 \times .4 \times .35 \times .7)]}=.593$. Thus, in this case the subject in position 4 should ignore his or her private signal and join the established pattern of predictions. Once he or she enters a cascade, subjects in later than position 4 should ignore their private signals of $\sigma_{B}$ as well.

In seniority, imbalances between Bayesian posterior probability and private signal occur as many as at 110 out of 252 decision nodes. More importantly, the subject in position 2 should make the same predictions as the subject in position 1 even when the private signal of the subject in position 2 does not correspond to the prediction of the subject in position 1 since $\operatorname{Pr}\left(A \mid \pi_{A}^{1}, \sigma_{B}^{2}\right)=\operatorname{Pr}\left(B \mid \pi_{B}^{1}, \sigma_{A}^{2}\right)=\frac{\frac{1}{2} \times .8 \times .25}{\frac{1}{2} \times[(.8 \times .25)+(.2 \times .75)]}=.571$. This leads to the result that subjects in a round should always make identical predictions whether they enter informational cascades or they truthfully reveal their private signals.

\section{$3 \quad$ Experimental Procedure}

Subjects were recruited from undergraduate students of Keio University, Shonan Fujisawa Campus. Six subjects participated in one session and they played the game for 16 rounds. Each session took less than 100 minutes including instruction and final questionnaire. Upon arriving the classroom, each subject drew a card for determining where to be seated. Each seat was separated and partitioned so that anyone could see neither the other subject's private draw nor his or her record sheet. Before each session began, subjects were instructed about the entire structure of the experiment. They were informed about their tasks, two sources of information they receive, combinations of signal quality and order of decisions, difference of each treatment, payoff scheme, and other procedures concerning the experiment in order to make them common knowledge among all subjects ${ }^{5}$. After instructions, subjects took a quiz about the basic structure of the experiment.

\footnotetext{
${ }^{3}$ For the list of Bayesian posterior probability at each decision node, see Appendix C.

${ }^{4}$ For the definition of complete informational cascades, see section 4.1 .

${ }^{5}$ Translated version of instructions for subjects is attached in Appendix B.
} 
If all the subjects submitted the correct answers, the experimental session began from the first round. At the beginning of each round, the experimenter announced which treatment to be conducted. Next, the experimenter drew one of the two cards from the covered box. On a card a letter either "A" or "B" was printed and the letter on the card drawn represented the state of the world for that round. The experimenter confirmed the letter so as not to be seen by subjects and hid it from them until the round finished. Then, each subject drew one of the six cards for determining the combinations of the signal quality and the order of decision. Since each subject had been notified which treatment would be conducted at the beginning of the round, there was no uncertainty in the combinations of signal quality and order of decisions for each round.

As in the procedure of Anderson and Holt's experiment, private signals were implemented by having subjects draw one of the twenty white and red marbles from a covered box. Marbles were identical in size, weight, and the feel of material. White marbles represented state A and red marbles represented state B. Different signal qualities were created by varying the proportion of white and red marbles in a box given the realized state, treatment, and the assigned signal quality for each subject. For example, if state A had realized, a subject who was assigned to position 1 in anti-seniority treatment drew a marble from the box containing 11 white and 9 red marbles. Combinations of the marbles in each position and state for each treatment is summarized in tables 2 and 3. Note that results of the draw indicate the correct state more accurately than random guessing even for the least precise quality of signal because the proportion of the white and red marbles correlates to the realized state.

A set of six boxes was used in one round and they are also identical in size, shape, and material except codes marked on the bottom of each boxes. They were stacked in a larger box separately by the states and were kept in the other room. After confirming the state for the round one of the experimenters brought the appropriate stack from that room so that subjects could not identify which stack was actually used.

By checking the codes on the bottom, the experimenter approached a subject one by one and presented one box which contained the exact proportion of white and red marbles for each subject. After confirming the color of the marble drawn, each subject replaced it into the box and wrote down the state indicated by the color in the space printed "Your Signal" on the subject's record sheet. Then, each subject was requested to make a prediction in the determined order. Subjects made a prediction by writing down one of the two states he or she thought which is more likely to be in the space printed "Your Prediction" on the subject's record sheet. The experimenter checked 
the prediction and wrote it down on the experimenter's record sheet. Then, the experimenter approached the subject in the next position and showed the sequence of predictions made by his or her predecessors ${ }^{6}$. In making predictions, subjects in positions from 2 to 6 can refer to them as well as their private signals whereas the subject in position 1 can observe only his or her private signal. After all six subjects submitted predictions, the card the experimenter had drawn at the beginning of the round was revealed, then subjects and the experimenter wrote down the results of their predictions. This process was repeated for 16 times with combinations of each treatment. The actual order of treatment in each session is shown in table $4^{7}$.

A total of 66 subjects participated in the experiments. After all 16 rounds finished subjects were paid payoffs privately in cash depending on the performance in a session. For each correct prediction 200 Japanese yen (eq. to $\$ 1.84$ ) were paid. Average payment for AA (anti-seniority, anti-seniority) treatments, AS (anti-seniority, seniority) treatments, and SA (seniority, antiseniority) treatment were 2176 yen, 2276 yen, and 2476 yen respectively ${ }^{8}$. In addition, the minimum payment and the maximum payment were 800 yen and 3200yen respectively.

\section{Results}

\subsection{Aggregated Behavior}

In order to compare the property of each treatment, I examine the results based on the following four criteria.

\footnotetext{
${ }^{6}$ In Anderson and Holt's experiment, they announced predecessors' predictions to all subjects. Thus, subjects who had completed making predictions could observe predictions made by other subjects in later positions. In my experiment, the experimenter reported predecessors' predictions only to the subject who was in making prediction. This is because I intended not to have subjects count predictions in later positions and use them as a heuristic in following rounds.

${ }^{7}$ In session 1 , only 12 rounds in anti-seniority treatment were conducted due to overtime. In session 2, anti-seniority treatment was conducted for all 16 rounds. In sessions 3 and 4, anti-seniority treatment was conducted for the first 12 rounds followed by seniority treatment for the last 4 rounds. For sessions 4 to 11 , each treatment is conducted by turns for the first 12 rounds and the last 4 rounds.

${ }^{8}$ In another day participants in session 1 were paid additional 544 yen which is the average payment of 4-round anti-seniority treatment as compensation for the early close of the session.
} 


\section{Complete positive (negative) cascades}

A complete positive (negative) cascade denotes a pattern of behaviors such that there is at least one subject who ignores his or her private signal and all of the six subjects in a round make identical correct (incorrect) predictions. Among complete positive (negative) cascades, a pattern of behaviors such that all predictions are consistent with Bayesian posterior probability is called a rational complete positive (negative) cascade.

\section{Partial cascades}

A partial cascade denotes a pattern of behaviors such that there is at least one subject who ignores his or her private signal but another subject collapses the established identical predictions. As a result, predictions are not identical in a round.

\section{Full revelations}

A full revelation denotes a pattern of behaviors such that all of the subjects make predictions consistent with their private signals. An aggregated behavior is counted as a full revelation whether they are consistent with Bayesian posteriors or not, and whether all of the predictions are identical or not.

\section{Herds}

A herd denotes identical predictions in a round. This may include complete cascades and full revelations ${ }^{9}$. Smith, L. and P. N. Sørensen (2000) and Çelen, B. and S. Kariv (2004) emphasized that there is a significant difference between informational cascades and herds. Informational cascades occur when subjects ignore their private signals in making predictions, whereas herds may occur when subjects happen to make identical predictions, not necessarily ignoring their private signals.

I report the results as a list of observations.

\section{Observation 1}

Learning within each session was not observed.

If subjects learn a systematic way of making predictions by some reasons, patterns of predictions should be different between earlier rounds and later rounds within a session. Tables 5 and 6 report the Mann-Whitney U

\footnotetext{
${ }^{9}$ Note that the other three criteria except herds do not overlap each other.
} 
test on equal proportion of predictions which is consistent with the Bayesian posterior probability between the first two rounds and the last two rounds within each session for each treatment. In anti-seniority, the null hypotheses of no difference cannot be rejected for all sessions. In seniority, almost all predictions in the first and the last two rounds within each session are identically consistent with Bayesian posterior so that the Mann-Whitney U test cannot be performed for those sessions. In sessions 6 and 11 where different proportions of Bayesian consistent predictions are observed, they are not significantly different between the first and last two rounds. Therefore, we can say that systematic pattern of learning within each session is not observed, so that we can pool the data for each treatment and compare the property of them. Results of the aggregated behavior in all of the 11 sessions for each treatment are summarized in table 7 .

\section{Observation 2}

Each state realized equally likely within and between each treatment.

The realizations of state $\mathrm{A}$ are 55 out of 112 rounds in anti-seniority and 31 out of 60 rounds in seniority (table 7 , row 1 ). The two-tailed test of population proportion cannot reject the null hypothesis that each state realized with equal frequency for each treatment (table 9). In addition, MannWhitney $U$ test shows that the realizations of state A are not significantly different between two treatments (table 8 row1). Therefore, draws by the experimenters are not biased within and between each treatment.

Observation 3

Subjects made more correct predictions in seniority than in anti-seniority.

Proportion of correct predictions in anti-seniority is $69.49 \%$ whereas that in seniority is $81.94 \%$ (table 7 , row 2). Mann-Whitney U test shows this difference is statistically significant (table 8 row 2 ). We can say that seniority increases the subjects' welfare in the sense that it leads to more correct predictions.

Observation 4

Complete (positive and negative) cascades and rational complete (positive and negative) cascades occurred more frequently in seniority than in antiseniority, whereas partial cascades occurred more frequently in anti-seniority than in seniority.

The proportion of complete positive cascades in anti-seniority is $14.29 \%$ whereas that in seniority is $60.00 \%$ (table 7 , row 3), which is significantly 
different (table 8, row 3). The proportion of complete negative cascades in anti-seniority is $1.79 \%$ whereas that in seniority is $10.00 \%$ (table 7 , row 4 ), which is also significantly different (table 8 row 4 ). The proportion of rational complete positive cascades in anti-seniority is $6.25 \%$ whereas that in seniority is $60.00 \%$ (table 7 , row 5 ), which is significantly different (table 8 , row 5). The proportion of rational complete negative cascades in antiseniority is $.89 \%$ whereas that in seniority is $10.00 \%$ (table 7 , row 6 ), which is also significantly different (table 8 , row 6 ). In addition, the proportion of partial cascades in anti-seniority is $25.89 \%$ whereas that in seniority is $10.00 \%$ (table 7 , row 7 ), which is significantly different (table 8 row 7 ). Therefore seniority enhances the occurrence of complete positive cascades but it also increases the risk for creating complete negative cascades. Meanwhile, the established cascades are more likely to be collapsed in anti-seniority than in seniority.

\section{Observation 5}

For both treatments, rational complete (positive and negative) cascades occurred less frequently than those which the Bayesian theory predicts.

For anti-seniority, rational complete cascades occur if the first three subjects make the same predictions as seen in section 2. By Bayes' rule, if the first three consecutive subjects made same predictions, the fourth subject cannot help making the same prediction as them even when his or her private signal does not correspond to the predecessors' predictions. Since herds by full revelations are not counted in rational complete positive cascades, the probability that rational complete positive cascades occur is given by the probability that the all six subjects draw the correct marbles subtracted from the probability that the first three subjects draw the correct marbles. That is, $\left[\operatorname{Pr}\left(A \mid \sigma_{A}^{1}\right) \times \operatorname{Pr}\left(A \mid \sigma_{A}^{2}\right) \times \operatorname{Pr}\left(A \mid \sigma_{A}^{3}\right)\right]-\left[\operatorname{Pr}\left(A \mid \sigma_{A}^{1}\right) \times \operatorname{Pr}\left(A \mid \sigma_{A}^{2}\right) \times \operatorname{Pr}\left(A \mid \sigma_{A}^{3}\right) \times\right.$ $\left.\operatorname{Pr}\left(A \mid \sigma_{A}^{4}\right) \times \operatorname{Pr}\left(A \mid \sigma_{A}^{5}\right) \times \operatorname{Pr}\left(A \mid \sigma_{A}^{6}\right)\right]=\left[\operatorname{Pr}\left(B \mid \sigma_{B}^{1}\right) \times \operatorname{Pr}\left(B \mid \sigma_{B}^{2}\right) \times \operatorname{Pr}\left(B \mid \sigma_{B}^{3}\right)\right]-$ $\left[\operatorname{Pr}\left(B \mid \sigma_{B}^{1}\right) \times \operatorname{Pr}\left(B \mid \sigma_{B}^{2}\right) \times \operatorname{Pr}\left(B \mid \sigma_{B}^{3}\right) \operatorname{Pr}\left(B \mid \sigma_{B}^{4}\right) \times \operatorname{Pr}\left(B \mid \sigma_{B}^{5}\right) \times \operatorname{Pr}\left(B \mid \sigma_{B}^{6}\right)\right]=$ $(.55 \times .6 \times .65)-(.55 \times .6 \times .65 \times .7 \times .75 \times .8)=.215-.090=.125$ On the other hand, rational complete negative cascades would occur if all of the first three subjects happen to make incorrect predictions. The probability that rational complete negative cascades occur is given by the probability that the all six subjects draw the incorrect marbles subtracted from the probability that the first three subjects draw the incorrect marbles. That is, $\left[\operatorname{Pr}\left(A \mid \sigma_{B}^{1}\right) \times \operatorname{Pr}\left(A \mid \sigma_{B}^{2}\right) \times \operatorname{Pr}\left(A \mid \sigma_{B}^{3}\right)\right]-\left[\operatorname{Pr}\left(A \mid \sigma_{B}^{1}\right) \times \operatorname{Pr}\left(A \mid \sigma_{B}^{2}\right) \times \operatorname{Pr}\left(A \mid \sigma_{B}^{3}\right) \times\right.$ $\left.\operatorname{Pr}\left(A \mid \sigma_{B}^{4}\right) \times \operatorname{Pr}\left(A \mid \sigma_{B}^{5}\right) \times \operatorname{Pr}\left(A \mid \sigma_{B}^{6}\right)\right]=\left[\operatorname{Pr}\left(B \mid \sigma_{A}^{1}\right) \times \operatorname{Pr}\left(B \mid \sigma_{A}^{2}\right) \times \operatorname{Pr}\left(B \mid \sigma_{A}^{3}\right)\right]-$ $\left[\operatorname{Pr}\left(B \mid \sigma_{A}^{1}\right) \times \operatorname{Pr}\left(B \mid \sigma_{A}^{2}\right) \times \operatorname{Pr}\left(B \mid \sigma_{A}^{3}\right) \operatorname{Pr}\left(B \mid \sigma_{A}^{4}\right) \times \operatorname{Pr}\left(B \mid \sigma_{A}^{5}\right) \times \operatorname{Pr}\left(B \mid \sigma_{A}^{6}\right)\right]=(.45 \times$ $.4 \times .35)-(.45 \times .4 \times .35 \times .3 \times .25 \times .2)=.063-.001=.062$. For seniority, all 
the subjects later than position 2 should follow the first subject's prediction, which inevitably leads to a herd in each round. Among rational complete cascades, whether they turn to be positive or negative depends exactly on whether the first subject makes the correct prediction. As in the case of anti-seniority, the probability that rational complete positive cascades occur is given by the probability that the all six subjects draw the correct marbles subtracted from the probability that the first subject draw the correct marble. That is, $\operatorname{Pr}\left(A \mid \sigma_{A}^{1}\right)-\left[\operatorname{Pr}\left(A \mid \sigma_{A}^{1}\right) \times \operatorname{Pr}\left(A \mid \sigma_{A}^{2}\right) \times \operatorname{Pr}\left(A \mid \sigma_{A}^{3}\right) \times \operatorname{Pr}\left(A \mid \sigma_{A}^{4}\right) \times \operatorname{Pr}\left(A \mid \sigma_{A}^{5}\right) \times\right.$ $\left.\operatorname{Pr}\left(A \mid \sigma_{A}^{6}\right)\right]=\operatorname{Pr}\left(B \mid \sigma_{B}^{1}\right)-\left[\operatorname{Pr}\left(B \mid \sigma_{B}^{1}\right) \times \operatorname{Pr}\left(B \mid \sigma_{B}^{2}\right) \times \operatorname{Pr}\left(B \mid \sigma_{B}^{3}\right) \operatorname{Pr}\left(B \mid \sigma_{B}^{4}\right) \times\right.$ $\left.\operatorname{Pr}\left(B \mid \sigma_{B}^{5}\right) \times \operatorname{Pr}\left(B \mid \sigma_{B}^{6}\right)\right]=(.8)-(.55 \times .6 \times .65 \times .7 \times .75 \times .8)=.8-.090=.710$. On the other hand, the probability that rational complete negative cascades occur is given by the probability that the all six subjects draw the incorrect marbles subtracted from the probability that the first subject draw the incorrect marble. That is, $\operatorname{Pr}\left(A \mid \sigma_{B}^{1}\right)-\left[\operatorname{Pr}\left(A \mid \sigma_{B}^{1}\right) \times \operatorname{Pr}\left(A \mid \sigma_{B}^{2}\right) \times \operatorname{Pr}\left(A \mid \sigma_{B}^{3}\right) \times\right.$ $\left.\operatorname{Pr}\left(A \mid \sigma_{B}^{4}\right) \times \operatorname{Pr}\left(A \mid \sigma_{B}^{5}\right) \times \operatorname{Pr}\left(A \mid \sigma_{B}^{6}\right)\right]=\operatorname{Pr}\left(B \mid \sigma_{A}^{1}\right)-\left[\operatorname{Pr}\left(B \mid \sigma_{A}^{1}\right) \times \operatorname{Pr}\left(B \mid \sigma_{A}^{2}\right) \times\right.$ $\left.\operatorname{Pr}\left(B \mid \sigma_{A}^{3}\right) \operatorname{Pr}\left(B \mid \sigma_{A}^{4}\right) \times \operatorname{Pr}\left(B \mid \sigma_{A}^{5}\right) \times \operatorname{Pr}\left(B \mid \sigma_{A}^{6}\right)\right]=(.2)-(.45 \times .4 \times .35 \times .3 \times$ $.25 \times .2)=.2-.001=.199$.

However, if we look at the proportions of rational complete cascades actually observed in experiments, they are consistently lower than those which the Bayesian theory predicts (table 7 , rows 5 and 6 ). The one-tailed test of population proportion shows that, for both treatments, the observed proportion of rational complete (positive and negative) cascades is significantly lower than the theoretical predictions (tables 10 and 11). This finding is consistent with previous experiments where fewer occurrences of informational cascades than the theoretical predictions were observed such as in Anderson, L. R. and C. A. Holt (1997), Çelen, B. and S. Kariv (2004), Huck, S. and J. Oechssler (2000), Nöth, M. and M. Weber (2000), and Oberhammer, C. and A. Stiehler (2001).

\section{Observation 6}

Full revelations occurred more frequently in anti-seniority than in seniority.

The proportion of full revelations in anti-seniority is $58.04 \%$ whereas that in seniority is $20.00 \%$ (table 7 , row 8 ), which is significantly different (table 8 , row 8 ). Thus, anti-seniority extracts private signals more effectively than seniority ${ }^{10}$.

\footnotetext{
${ }^{10}$ Ottaviani, M. and P. N. Sørensen (2001) studied theoretical model of informational cascades resulted from reputational concern in the framework of heterogeneous signal qualities. They showed that anti-seniority is effective in extracting private information in some circumstances, but is not always so.
} 


\section{Observation 7}

Herds occurred more frequently in seniority than in anti-seniority.

Herds are caused by complete cascades or full revelations. The proportion of herds in anti-seniority is $28.57 \%$ whereas that in seniority is $83.33 \%$ (table 7 row 9 ), which is significantly different (table 8 , row 9).

\subsection{Individual Behavior}

We observed that aggregated behavior is different in several aspects between two treatments. Now let us examine how each subject uses the two sources of information, private signal and predecessors' predictions. In order to investigate how weight subjects place on the two sources of information, I use Grether,M. (1989) and Hung, A. and C. Plott (2000)'s logit regression model.

Let $\frac{\operatorname{Pr}\left(A \mid \pi_{\omega}^{1}, \ldots, \pi_{\omega}^{i-1}, \sigma_{\omega}^{i}\right)}{\operatorname{Pr}\left(B \mid \pi_{\omega}^{1}, \ldots, \pi_{\omega}^{i-1}, \sigma_{\omega}^{i}\right)}$ be the odds of the individual who are in position $i$ in favor of state A over state B after observing his or her predecessors' predictions $\pi_{\omega}^{1}, \ldots, \pi_{\omega}^{i-1}$ and his or her own private signal $\sigma_{\omega}^{i}$. Then,

$$
\begin{aligned}
\frac{\operatorname{Pr}\left(A \mid \pi_{\omega}^{1}, \ldots, \pi_{\omega}^{i-1}, \sigma_{\omega}^{i}\right)}{\operatorname{Pr}\left(B \mid \pi_{\omega}^{1}, \ldots, \pi_{\omega}^{i-1}, \sigma_{\omega}^{i}\right)} & =\frac{\operatorname{Pr}(A) \operatorname{Pr}\left(\pi_{\omega}^{1}, \ldots, \pi_{\omega}^{i-1}, \sigma_{\omega}^{i} \mid A\right) / \operatorname{Pr}\left(\pi_{\omega}^{1}, \ldots, \pi_{\omega}^{i-1}, \sigma_{\omega}^{i}\right)}{\operatorname{Pr}(B) \operatorname{Pr}\left(\pi_{\omega}^{1}, \ldots, \pi_{\omega}^{i-1}, \sigma_{\omega}^{i} \mid B\right) / \operatorname{Pr}\left(\pi_{\omega}^{1}, \ldots, \pi_{\omega}^{i-1}, \sigma_{\omega}^{i}\right)} \\
& =\frac{\operatorname{Pr}(A) \operatorname{Pr}\left(\pi_{\omega}^{1}, \ldots, \pi_{\omega}^{i-1}, \sigma_{\omega}^{i} \mid A\right)}{\operatorname{Pr}(B) \operatorname{Pr}\left(\pi_{\omega}^{1}, \ldots, \pi_{\omega}^{i-1}, \sigma_{\omega}^{i} \mid B\right)} \\
& =\frac{\operatorname{Pr}(A) \operatorname{Pr}\left(\pi_{\omega}^{1}, \ldots, \pi_{\omega}^{i-1} \mid A\right) \operatorname{Pr}\left(\sigma_{\omega}^{i} \mid A\right)}{\operatorname{Pr}(B) \operatorname{Pr}\left(\pi_{\omega}^{1}, \ldots, \pi_{\omega}^{i-1} \mid B\right) \operatorname{Pr}\left(\sigma_{\omega}^{i} \mid B\right)} .
\end{aligned}
$$

The last equation holds because of the assumption that $\pi_{\omega}^{1}, \ldots, \pi_{\omega}^{i-1}$ and $\sigma_{\omega}^{i}$ are independent. By taking logs,

$$
\ln \frac{\operatorname{Pr}\left(A \mid \pi_{\omega}^{1}, \ldots, \pi_{\omega}^{i-1}, \sigma_{\omega}^{i}\right)}{\operatorname{Pr}\left(B \mid \pi_{\omega}^{1}, \ldots, \pi_{\omega}^{i-1}, \sigma_{\omega}^{i}\right)}=\ln \frac{\operatorname{Pr}(A)}{\operatorname{Pr}(B)}+\ln \frac{\operatorname{Pr}\left(\pi_{\omega}^{1}, \ldots, \pi_{\omega}^{i-1} \mid A\right)}{\operatorname{Pr}\left(\pi_{\omega}^{1}, \ldots, \pi_{\omega}^{i-1} \mid B\right)}+\ln \frac{\operatorname{Pr}\left(\sigma_{\omega}^{i} \mid A\right)}{\operatorname{Pr}\left(\sigma_{\omega}^{i} \mid B\right)}
$$

Since $\ln \frac{\operatorname{Pr}(A)}{\operatorname{Pr}(B)}=0,(1)$ can be generalized to

$$
Y_{m}^{i}=\beta_{0}+\beta_{1} \ln \frac{\operatorname{Pr}\left(\pi_{\omega}^{1}, \ldots, \pi_{\omega}^{i-1} \mid A\right)}{\operatorname{Pr}\left(\pi_{\omega}^{1}, \ldots, \pi_{\omega}^{i-1} \mid B\right)}+\beta_{2} \ln \frac{\operatorname{Pr}\left(\sigma_{\omega}^{i} \mid A\right)}{\operatorname{Pr}\left(\sigma_{\omega}^{i} \mid B\right)}+u_{m}^{i}
$$

where $u_{m}^{i}$ is a random disturbance.

Three possible combinations of coefficients are of interest as special cases of equation (2). If $\beta_{0}=0$ and $\beta_{1}=\beta_{2}$, then subjects put equal weight on private signals and on predecessors' predictions. If $\beta_{0}=0$ and $0<\beta_{2}<\beta_{1}$, 
then subjects put more weight on predecessors' predictions than on private signals. If $\beta_{0}=0$ and $0<\beta_{1}<\beta_{2}$, then subjects put more weight on private signals than on predecessors' predictions. I estimate the coefficients of equation (2) by the logit regression for each treatment. The logit regression is performed for subjects in positions from 2 to 6 since the subjects in position 1 do not observe predecessors' predictions. The results of the logit regressions are shown in tables 12 and 13.

\section{Observation 8}

There is no systematic bias in predicting a state.

In both treatments $\beta_{0}=0$ cannot be rejected at the $95 \%$ significant level. Thus, predictions made by subjects are not biased toward one of the states.

Tables 14 and 15 show the chi-square tests of the null hypothesis that the estimated coefficients of private signals and predecessors' predictions are equal. This hypothesis can be rejected for anti-seniority whereas cannot be rejected for seniority. To summarize, we can conclude that

\section{Observation 9}

Subjects in seniority put equal weight on private signals and on predecessors' predictions whereas subjects in anti-seniority put more weight on private signals than on predecessors' predictions.

\subsection{Analysis of Deviation}

We observed that rational complete cascades occur significantly less frequently than those which the Bayesian theory predicts for both treatments. Then, we should investigate why human subjects deviate from the prediction of the Bayesian theory. To do so, we formally define three types of deviations, overconfidence, underconfidence, and irrational as often emphasized in probabilistic inference problem (Edwards, W. (1968), Griffin, D. and A. Tversky (1992), Tversky, A. and D. Kahneman (1971), Weinstein, N. (1980)). Overconfidence can be defined as a prediction which is not consistent with Bayesian posterior but consistent with the subject's private signal. Underconfidence can be defined as a prediction which is consistent with neither Bayesian posterior nor the subject's private signal but consistent with a prediction which is made by at least one of his or her predecessors. Deviations which is not consistent with Bayesian posterior and cannot be explained by these two criteria are defined as irrational ${ }^{11}$.

\footnotetext{
${ }^{11}$ Therefore, an irrational prediction is consistent neither with Bayesian posterior, the subject's private signal, nor any predecessors' predictions
} 
The proportions of overconfidence, underconfidence, and irrational for each treatment and for each position are summarized in tables 16 and 17 and depicted in figures 1 and 2. In anti-seniority, overconfidence cannot occur in positions 2 and 3 because obeying private signals is always consistent with the Bayesian posteriors ${ }^{12}$. In seniority, underconfidence cannot occur in position 2 because obeying the prediction of the subject in position 1 and ignoring his or her private signal is always consistent with Bayesian posterior. Overconfidence and underconfidence certainly exist in anti-seniority whereas only overconfidence occurred frequently in position 2 and deviations are rarely observed in the other positions in seniority ${ }^{13}$.

Given the existence of overconfidence and underconfidence, one might think that subjects' predictions are "anchored" by their signal qualities ${ }^{14}$. That is, subjects with more precise signals tend to be overconfident and subjects with less precise signals tend to be underconfident. To examine this, we need to investigate the relationship between qualities of private signals and deviating behaviors for each Bayesian posterior probability. Tables 18-21 show that the percentage of deviations, for each position, categorized by five levels of Bayesian posteriors that the state A would have realized. Note that deviations should occur most frequently in the level of Bayesian posterior $40-60 \%$ because subjects would not be sure which state has realized around posteriors $50 \%$.

If there exists the anchoring effect in anti-seniority, deviation by underconfidence would occur more in earlier positions with more broad range of posteriors and less in later positions with less broad range of posteriors because subjects with less precise signals are underconfident too much about

\footnotetext{
${ }^{12}$ For the list of Bayesian posterior probability in each decision node, see Appendix C.

${ }^{13}$ The existence of underconfidence would create possibility of cascades whereas the existence of overconfidence would collapse potential cascades. If there is at least one subject who makes an overconfident prediction, the potential cascades will inevitably collapse. The fact that rational complete cascades are observed less frequently than Bayesian theory predicts can be attributed to the existence of overconfidence. This observation that existence of overconfidence reduce the cascades is consistent with past several experiments, such as Kraemer, T. and M. Nöth (2000) and Nöth, M. and M. Weber (2000).

${ }^{14}$ Kraemer, T. and M. Nöth (2000) reported that they identified the anchoring effect. In their experiment they compared posteriors evaluated by subjects and actual posteriors. By calculating their differences, they found that they are minimized when the posteriors are close to subjects' private signals and concluded that this fact supports anchoring hypothesis. However, the problem is that they did not provide subjects with any incentive to evaluate posteriors seriously as Oberhammer, C. and A. Stiehler (2001) pointed out. In order to investigate existence of the anchoring effect, one needs to look at deviating behavior itself resulting from over- and underconfidence and examine the relationship between deviation and signal quality for each Bayesian posteriors. The following part of this section tries to do it.
} 
the realized state compared to the actual Bayesian posteriors. On the other hand, deviations by overconfidence would occur more in later positions with more broad range of posteriors and less in earlier positions with less broad range of posteriors because subjects with more precise signals are confident too much about the realized state compared to the actual Bayesian posteriors.

Observed deviations by underconfidence in anti-seniority (table 18) certainly decrease from $9.82 \%$ in position 3 to $1.78 \%$ in position 5 . However, there are two increases from $4.47 \%$ in position 2 to $9.82 \%$ in position 3 and from $1.78 \%$ in position 5 to $2.68 \%$ in position 6 . In addition, deviations except in the range of $40-60 \%$ occurred not in the earlier positions but in the later positions. These two observations are not consistent with the systematic pattern of deviations discussed above so that we conclude that the anchoring hypothesis has only a partial explanation for deviations by underconfidence in anti-seniority. For observed deviations by overconfidence in anti-seniority (table 19), they increase from $5.36 \%$ in position 4 to $12.50 \%$ in position 6 and deviations except 40\%-60\% occurred not in the earlier positions but in the later positions. In position 5 , some deviations occurred in the level of posteriors $20-40 \%$ or $60-80 \%$. Furthermore, in position 6 , deviations occurred even when Bayesian posteriors suggest the realization of one state rather than the other with high probability such as in the level of posteriors $0-20 \%$ or $80-100 \%$. In fact, there were subjects who predicted "A" in spite that their posteriors that the state A would have realized were as low as $20 \%$ and those who predicted "B" in spite that their posteriors were as high as $85 \%$. By looking at deviations of positions $4-6$, we confirm that subjects with high signal qualities are more likely to deviate from the Bayesian posteriors. This systematic pattern of deviations, unlike the case of underconfidence, supports the hypothesis of the anchoring effect.

If there exists the anchoring effect in seniority, by the same reasoning as in anti-seniority, deviations by underconfidence would occur more in later positions with more broad range of posteriors and less in earlier positions with less broad range of posteriors. On the other hand, deviations by overconfidence would occur more in earlier positions with more broad range of posteriors and less in later positions with less broad range of posteriors.

However, we observed seldom deviations in seniority as the results of logit regression suggest. Obvious deviations occurred only in position 2 for overconfidence when Bayesian posteriors are either $42.8 \%$ or $57.2 \%$. This fact moderately supports the hypothesis of the anchoring effect for overconfidence, however few observations of deviations for underconfidence do not support it for underconfidence. To summarize, we have the following observation. 


\section{Observation 10}

The anchoring effect is identified in deviations by overconfidence but not identified in deviations by underconfidence.

Why is not the anchoring effect clearly identified in deviations by underconfidence in both treatments?

A possible reason for unidentifiability of the anchoring effect in seniority can be attributed to the intrinsic structure of posteriors in seniority. Since posteriors that state A would have realized are extremely high or low in later positions such as 5 or 6 in seniority, subjects with less precise signals can predict consistent with Bayesian posteriors relatively easily by following the established pattern of predecessors' predictions even when their private signals do not correspond to them. In such cases, making underconfident predictions are almost irrational.

A possible reason for the unclear identification of the anchoring effect in anti-seniority would be related to "the strength and weight effect of the sample". Griffin, D. and A. Tversky (1992) studied this effect in determining subject's attitudes toward overconfidence and underconfidence. Through a series of hypothetical coin-spinning experiments, they found that human subjects are more likely to be overconfident when the sample is small and it shows extreme result (i.e. weight of the sample is low and strength of the sample is high), and more likely to be underconfident when the sample is large and it shows moderate result (i.e. weight of the sample is high and strength of the sample is low $)^{15}$.

If the strength and weight effect of the sample exists in our informational cascades experiment, subjects in earlier positions would be overconfident whereas subjects in later positions would be underconfident because subjects in earlier positions have a smaller sample (i.e. fewer predecessors' predictions) than subjects in later positions ${ }^{16}$. Then, underconfidence in earlier positions would be offset by this overconfidence and result in the unclear identification of the anchoring effect in anti-seniority.

\footnotetext{
${ }^{15}$ In probabilistic inference experiments, Edwards, W. (1968) observed that subjects were more likely to be underconfident whereas Tversky, A. and D. Kahneman (1971) verified that they were more likely to be overconfident compared to the Bayesian posterior. However, Griffin, D. and A. Tversky (1992) argued that these inconsistent observations are comprehensible as the former used a large and moderately strong sample and the latter used a small and fairly strong sample.

${ }^{16}$ Overconfidence which would be caused by the strength and weight effect of the sample is observed in informational cascades experiment by Çelen, B. and S. Kariv (2004). They found that "subjects who are early decision-makers tend to rely more heavily on their own information in the learning process, which then becomes available to late decision-makers, who tend more to be Bayesian."
} 
However, if the above explanation holds, we have to say that this experiment confounds the anchoring effect and the strength and weight effect of the sample. Although the anchoring effect is identified in deviations by overconfidence in anti-seniority this should have been also canceled out by underconfidence resulted from the strength and weight effect of the sample to some extent. In order to evaluate each effect clearly, we need to distinguish each other and investigate how these two effects interact in various situations. Because this experiment cannot do that, we need some other settings. One possible improvement would be in a design of an experiment where signal quality is identical in one treatment but is different among different treatments. By comparing patterns of deviations resulted from different treatments, one would be able to identify the anchoring effect and the strength and weights effect more clearly.

\section{Conclusion}

By implementing heterogenous signal qualities associated with the fixed orders of making predictions in the two different decision-making systems, we have observed the different consequences of each system and identified heuristics human subjects use.

For the investigations of anti-seniority and seniority, we have observed the following. Complete cascades occur more frequently in seniority than in antiseniority. Seniority is more efficient than anti-seniority, but it increases the risk for creating negative cascades. For both treatments, rational complete cascades occur less frequently than those which the Bayesian theory predicts. Private signals can be extracted more effectively in anti-seniority than in seniority. In the process of decision-making, subjects in seniority put equal weight on private signals and on predecessors' predictions whereas subjects in anti-seniority put more weight on private signals than on predecessors' predictions.

By analyzing the relationship between signal qualities and deviations from Bayesian posteriors, we have identified both overconfidence and underconfidence. In addition, the anchoring effect is verified in deviations by overconfidence, but is not verified in deviations by underconfidence. The unidentification problem of the anchoring effect in deviations by underconfidence in seniority would be due to the intrinsic structure of posteriors where subjects in later positions consistently face extreme high or low posteriors. The unclear identification problem of the anchoring effect in deviations by underconfidence in anti-seniority can be explained by the strength and weight effect of the sample by which subjects with the small sample would be over- 
confident. In order to investigate the relationship between the anchoring effect and the strength and weight effect of the sample more accurately, we have to differentiate each other in some other experimental settings. 


\section{References}

[1] Anderson, L. R. and C. A. Holt, "Information Cascades in the Laboratory," American Economic Review, 1997, 87 (5), 847-62.

[2] Bannerjee, A. V., "A Simple Model of Herd Behavior," Quarterly Journal of Economics, 1992, 107 (3), 797-817.

[3] Çelen, B. and S. Kariv, "Distinguishing Informational Cascades from Herd Behavior in the Laboratory," American Economic Review, 2004, 94 (3), 484-98.

[4] Edwards, W., "Conservatism in Human Information Processing," in B. Kleinmuntz (Ed.), Formal Representation of Human Judgement, 1968, 17-52, New York, Wiley.

[5] Grether, M., "Bayes Rule as a Descriptive Model," Quarterly Journal of Economics, 1980, 95 (3), 537-57.

[6] Griffin, D. and A. Tversky, "The Weighing of Evidence and the Determinants of Confidence," Cognitive Psychology, 1992, 24 (3), 411-35.

[7] Huck, S. and J. Oechssler, "Informational Cascades in the Laboratory: Do They Occur for the Right Reasons?, " Journal of Economic Psychology, 2000, 21 (6), 661-71.

[8] Hung, A A. and C. R. Plott, "Information Cascades: Replication and an Extension to Majority Rule and Conformity Rewarding Institutions," American Economic Review, 2001, 91 (5), 1508-20.

[9] Kraemer, T. and M. Nöth, "Anchoring and Adjustment in Information Cascades: Experimental Evidence," 2000, mimeo.

[10] Kraemer, C., M. Nöth, and M. Weber, "Information Aggregation with Costly Information and Random Ordering: Experimental Evidence," Sonder Forschungs Bereich 504 Working Paper Series 00-35, Universität Mannheim, 2000.

[11] Nöth, M. and M. Weber, "Information Aggregation with Random Ordering: Cascades and Overconfidence," Sonder Forschungs Bereich 504 Working Paper Series 00-34, Universität Mannheim, 2000.

[12] Oberhammer, C. and A. Stiehler, "Does Cascade Behavior in Information Cascades Reflect Bayesian Updating? - An Experimental Study -," Humboldt-University of Berlin, 2001.

[13] Ottaviani, M. and P. N. Sørensen, "Information Aggregation in debate: Who Should Speak First?," Journal of Public Economics, 2001, 81 (3), 393-421. 
[14] Smith, L. and P. N. Sørensen, "Pathological Outcomes of Observational Learning," Econometrica, 2000, 68 (2), 371-98.

[15] Tversky, A. and D. Kahneman, "The Belief in the Law of Small Numbers," Psychological Bulletin, 1971, 76, 105-110.

[16] Weinstein, N. D., "Unrealistic Optimism About Future Life Events," Journal of Personality and Social Psycology, 1980, 39, 806-20. 


\section{Appendices}

\section{A Tables and figures}

\begin{tabular}{|c|c|c|c|c|c|c|c|}
\hline \multicolumn{2}{|c|}{} & \multicolumn{6}{|c|}{ Position } \\
\hline \hline \multicolumn{2}{|c|}{ Treatment } & 1 & 2 & 3 & 4 & 5 & 6 \\
\cline { 2 - 9 } & Anti-seniority & .55 & .6 & .65 & .7 & .75 & .8 \\
\cline { 2 - 9 } & Seniority & .8 & .75 & .7 & .65 & .6 & .55 \\
\hline
\end{tabular}

Table 1: Combination of signal qualities and order of decisions in each treatment

\begin{tabular}{|c|c|c|c|c|c|c|c|}
\hline \multicolumn{9}{|c|}{ Anti-seniority } \\
\hline \hline \multicolumn{2}{|c|}{} & \multicolumn{6}{|c|}{ Position (signal quality) } \\
\hline $\begin{array}{c}\text { Realized } \\
\text { state }\end{array}$ & Marbles & $1(.55)$ & $2(.6)$ & $3(.65)$ & $4(.7)$ & $5(.75)$ & $6(.8)$ \\
\hline A & White & 11 & 12 & 13 & 14 & 15 & 16 \\
& Red & 9 & 8 & 7 & 6 & 5 & 4 \\
B & White & 9 & 8 & 7 & 6 & 5 & 4 \\
& Red & 11 & 12 & 13 & 14 & 15 & 16 \\
\hline
\end{tabular}

Table 2: Contents of boxes in each state and each position: Anti-seniority

\begin{tabular}{|c|c|c|c|c|c|c|c|}
\hline \multicolumn{9}{|c|}{ Seniority } \\
\hline \hline \multicolumn{2}{|c|}{} & \multicolumn{6}{|c|}{ Position (signal quality) } \\
\hline $\begin{array}{c}\text { Realized } \\
\text { state }\end{array}$ & Marbles & $1(.8)$ & $2(.75)$ & $3(7)$ & $4(.65)$ & $5(.6)$ & $6(.55)$ \\
\hline A & White & 16 & 15 & 14 & 13 & 12 & 11 \\
& Red & 4 & 5 & 6 & 7 & 8 & 9 \\
B & White & 4 & 5 & 6 & 7 & 8 & 9 \\
& Red & 16 & 15 & 14 & 13 & 12 & 11 \\
\hline
\end{tabular}

Table 3: Contents of boxes in each state and each position: Seniority

\begin{tabular}{|l|ccccccccccc|}
\hline Sessions & 1 & 2 & 3 & 4 & 5 & 6 & 7 & 8 & 9 & 10 & 11 \\
\hline \hline Rounds 1-12 & A & A & A & A & A & S & A & S & A & S & A \\
Rounds 12-16 & - & A & S & S & S & A & S & A & S & A & S \\
\hline
\end{tabular}

Table 4: Treatments conducted in each sessions

(A: Anti-seniority, S:Seniority) 


\begin{tabular}{|c|c|c|c|c|c|c|}
\hline \multicolumn{7}{|c|}{ Anti-seniority } \\
\hline \hline Session & 1 & 2 & 3 & 4 & 5 & 6 \\
\hline$z$ & -.440 & -.604 & .000 & 1.319 & -.604 & 1.813 \\
$P>|z|$ & .660 & .546 & 1.000 & .187 & .546 & .070 \\
\hline Session & 7 & 8 & 9 & 10 & 11 & \\
\cline { 1 - 5 } & -.604 & 1.000 & -1.000 & .000 & .604 & \\
$P>|z|$ & .546 & .3173 & .3173 & 1.000 & .546 & \\
\hline
\end{tabular}

Table 5: Mann-Whitney U tests of equal proportion of predictions which is consistent with Bayesian posterior probability between the first two rounds and the last two rounds for each session: Anti-seniority

\begin{tabular}{|c|c|c|}
\hline \multicolumn{3}{|c|}{ Seniority } \\
\hline \hline Session & 6 & 11 \\
\hline$z$ & 1.000 & 1.000 \\
$P>|z|$ & .3173 & .3173 \\
\hline
\end{tabular}

Table 6: Mann-Whitney U tests of equal proportion of predictions which is consistent with Bayesian posterior probability between the first two rounds and the last two rounds for each session: Seniority ${ }^{17}$

\begin{tabular}{|c|c|c|}
\hline \multicolumn{3}{|c|}{ Aggregated behavior } \\
\hline \hline & Anti-seniority & Seniority \\
& 112 rounds, & 60 rounds, \\
& 672 decisions & 360 decisions \\
\hline Realization of state A & $55(49.11 \%)$ & $31(51.67 \%)$ \\
Correct predictions & $467(69.49 \%)$ & $295(81.94 \%)$ \\
Complete positive cascades & $16(14.29 \%)$ & $36(60.00 \%)$ \\
Complete negative cascades & $2(1.79 \%)$ & $6(10.00 \%)$ \\
Rational complete positive cascades & $7(6.25 \%)$ & $36(60.00 \%)$ \\
Rational complete negative cascades & $1(.89 \%)$ & $6(10.00 \%)$ \\
Partial cascades & $29(25.89 \%)$ & $6(10.00 \%)$ \\
Full revelations & $65(58.04 \%)$ & $12(20.00 \%)$ \\
Herds & $32(28.57 \%)$ & $50(83.33 \%)$ \\
\hline
\end{tabular}

Table 7: Aggregated behavior

\footnotetext{
${ }^{17}$ All the predictions except in sessions 6 and 11 in the first and the last two rounds were identically consistent with Bayesian posterior probability, so that Mann-Whitney test of the equal proportions cannot be performed for those sessions.
} 


\begin{tabular}{|c|c|c|}
\hline \multicolumn{3}{|c|}{ Tests of equal proportion for each treatment } \\
\hline \hline & $z$ & $P>|z|$ \\
\hline Realization of state A & -.319 & .7497 \\
Correct predictions & -4.335 & .0000 \\
Complete positive cascades & -6.204 & .0000 \\
Complete negative cascades & -2.431 & .0151 \\
Rational complete positive cascades & -7.736 & .0000 \\
Rational complete negative cascades & -2.873 & .0041 \\
Partial cascades & 2.460 & .0139 \\
Full revelations & 4.767 & .0000 \\
Herds & -6.833 & .0000 \\
\hline
\end{tabular}

Table 8: Mann-Whitney U tests of equal proportion for each treatment

\begin{tabular}{|c|c|}
\hline \multicolumn{2}{|c|}{ Realization of each state } \\
\hline \hline Anti-seniority & Seniority \\
\hline $\mathrm{z}=-.189, P>|z|=.8501$ & $\mathrm{z}=.258, P>|z|=.7963$ \\
\hline
\end{tabular}

Table 9: Tests of population proportion on equal realization of each state

\begin{tabular}{|c|c|}
\hline $\begin{array}{c}\text { Theoretical prediction and actual occurrence: } \\
\text { Rational complete positive cascades }\end{array}$ \\
\hline \hline Anti-seniority & Seniority \\
\hline $\mathrm{z}=-2.000, P<z=.0228$ & $\mathrm{z}=-1.878, P<z=.0302$ \\
\hline
\end{tabular}

Table 10: Tests of population proportion on the equality between theoretical prediction and actual occurrence:

Rational complete positive cascades

\begin{tabular}{|c|c|}
\hline $\begin{array}{c}\text { Theoretical prediction and actual occurrence: } \\
\text { Rational complete negative cascades }\end{array}$ \\
\hline \hline Anti-seniority & Seniority \\
\hline $\mathrm{z}=-2.329, P<z=.0099$ & $\mathrm{z}=-1.921, P<z=.0274$ \\
\hline
\end{tabular}

Table 11: Tests of population proportion on the equality between theoretical prediction and actual occurrence:

Rational complete negative cascades 
Anti-seniority

\begin{tabular}{|c|c|c|c|c|}
\hline \hline \multicolumn{5}{|c|}{ Obs.: 560, Log-likelihood:-146.0963, $\chi^{2}: 483.95, P>\chi^{2}: .0000$, Pseudo $R^{2}: .6235$} \\
\hline & Estimated coefficient & Standard error & $z$ & $P>|z|$ \\
\hline$\beta_{0}$ & -.0946 & .1509 & -.63 & .531 \\
$\beta_{1}$ & 1.9674 & .2295 & 8.57 & .000 \\
$\beta_{2}$ & 3.6489 & .3212 & 11.36 & .000 \\
\hline
\end{tabular}

Table 12: Result of logit regression: Anti-seniority

\begin{tabular}{|c|c|c|c|c|}
\hline \multicolumn{4}{|c|}{ Seniority } \\
\hline \hline \multicolumn{4}{|c|}{ Obs.: 300, Log-likelihood:-32.1571, $\chi^{2}: 349.32 P>\chi^{2}: .0000$, Pseudo $R^{2}: .8445$} \\
\hline & Estimated coefficient & Standard error & $z$ & $P>|z|$ \\
\hline$\beta_{0}$ & -.6120 & .3603 & -1.70 & .089 \\
$\beta_{1}$ & 1.7908 & .2923 & 6.13 & .000 \\
$\beta_{2}$ & 2.4707 & .5487 & 4.50 & .000 \\
\hline
\end{tabular}

Table 13: Result of logit regression: Seniority

\begin{tabular}{|c|}
\hline Test of estimated coefficients: Anti-seniority \\
\hline \hline$H_{0}: \beta_{1}=\beta_{2}$ \\
$\chi^{2}: 75.72$ \\
$P>\chi^{2}: .0000$ \\
\hline
\end{tabular}

Table 14: Chi-square test of the hypothesis that estimated coefficients of private signals and predecessors' predictions are equal: Anti-seniority

\begin{tabular}{|c|}
\hline Test of estimated coefficients: Seniority \\
\hline \hline$H_{0}: \beta_{1}=\beta_{2}$ \\
$\chi^{2}: 3.02$ \\
$P>\chi^{2}: .0822$ \\
\hline
\end{tabular}

Table 15: Chi-square test of the hypothesis that estimated coefficients of private signals and predecessors' predictions are equal: Seniority 


\begin{tabular}{|c|c|c|c|}
\hline \multicolumn{4}{|c|}{ Deviations from Bayesian posterior by position } \\
\hline \hline Position & Overconfidence & Underconfidence & Irrational \\
\hline 1 & - & - & $15(13.39 \%)$ \\
2 & - & $5(4.47 \%)$ & $2(1.78 \%)$ \\
3 & - & $11(9.82 \%)$ & $0(.00 \%)$ \\
4 & $6(5.36 \%)$ & $4(3.57 \%)$ & $2(1.78 \%)$ \\
5 & $11(9.82 \%)$ & $2(1.78 \%)$ & $0(.00 \%)$ \\
6 & $14(12.50 \%)$ & $3(2.68 \%)$ & $0(.00 \%)$ \\
Total & $31(9.23 \% 18)$ & $25(4.46 \%)$ & $19(2.83 \%)$ \\
\hline
\end{tabular}

Table 16: Deviations from Bayesian posterior by position: Anti-seniority

\begin{tabular}{|c|c|c|c|}
\hline \multicolumn{4}{|c|}{ Deviations from Bayesian posterior by position } \\
\hline \hline Position & Overconfidence & Underconfidence & Irrational \\
\hline 1 & - & - & $0(.00 \%)$ \\
2 & $9(15.00 \%)$ & - & $0(.00 \%)$ \\
3 & $0(.00 \%)$ & $0(.00 \%)$ & $0(.00 \%)$ \\
4 & $0(.00 \%)$ & $1(1.67 \%)$ & $0(.00 \%)$ \\
5 & $1(1.67 \%)$ & $0(.00 \%)$ & $0(.00 \%)$ \\
6 & $0(.00 \%)$ & $0(.00 \%)$ & $0(.00 \%)$ \\
Total & $10(3.33 \%)$ & $1(.004 \%)$ & $0(.00 \%)$ \\
\hline
\end{tabular}

Table 17: Deviations from Bayesian posterior by position: Seniority

\begin{tabular}{|c|c|c|c|c|c|c|}
\hline \multicolumn{7}{|c|}{$\begin{array}{l}\text { Deviations from Bayesian posterior } \\
\text { by position and the level of posteriors }\end{array}$} \\
\hline & & \multicolumn{5}{|c|}{$\%$ of deviations by position } \\
\hline & & 2 & 3 & 4 & 5 & 6 \\
\hline Bayesian posterior & $0-20$ & .00 & .00 & .00 & 89 & .00 \\
\hline probabilities that state & $20-40$ & .00 & .00 & .89 & .00 & .89 \\
\hline \multirow[t]{3}{*}{ A would have realized (\%) } & $40-60$ & 4.47 & 9.82 & 1.79 & .00 & .89 \\
\hline & $60-80$ & .00 & .00 & .89 & .89 & .00 \\
\hline & $80-100$ & .00 & .00 & .00 & .00 & .89 \\
\hline
\end{tabular}

Table 18: Percentage of deviations from Bayesian posterior probability for each position and each level of posterior: Underconfidence in anti-seniority

\footnotetext{
${ }^{18}$ The percentage in the total deviations for tables 16 and 17 is all the observed deviations devided by total decisions where deviations are possible.
} 


\begin{tabular}{|c|c|c|c|c|}
\hline \multicolumn{5}{|c|}{ Deviations from Bayesian posterior } \\
by position and the level of posterior \\
\hline \hline \multicolumn{2}{|c|}{} & $\%$ of deviations by position \\
\cline { 3 - 5 } & 4 & 5 & 6 \\
\hline Bayesian posterior & $0-20$ & .00 & .00 & 2.68 \\
probabilities that state & $20-40$ & .00 & 2.68 & .00 \\
A would have realized (\%) & $40-60$ & 5.36 & 3.58 & 2.68 \\
& $60-80$ & .00 & 3.58 & 4.46 \\
& $80-100$ & .00 & .00 & 2.68 \\
\hline
\end{tabular}

Table 19: Percentage of deviations from Bayesian posterior probability for each position and each level of posterior: Overconfidence in anti-seniority

\begin{tabular}{|c|c|c|c|c|c|}
\hline \multicolumn{6}{|c|}{$\begin{array}{c}\text { Deviations from Bayesian posterior } \\
\text { by position and the level of posterior }\end{array}$} \\
\hline \hline \multicolumn{2}{|c|}{} & & $\%$ of deviations by position \\
\cline { 3 - 6 } & 3 & 4 & 5 & 6 \\
\hline Bayesian posterior & $0-20$ & .00 & .00 & .00 & .00 \\
probabilities that state & $20-40$ & .00 & .00 & .00 & .00 \\
A would have realized (\%) & $40-60$ & .00 & .00 & .00 & .00 \\
& $60-80$ & .00 & 1.79 & .00 & .00 \\
& $80-100$ & .00 & .00 & .00 & .00 \\
\hline
\end{tabular}

Table 20: Percentage of deviations from Bayesian posterior probability for each position and each level of posterior: Underconfidence in seniority

\begin{tabular}{|c|c|c|c|c|c|c|}
\hline \multicolumn{7}{|c|}{ Deviations from Bayesian posterior } \\
by position and the level of posterior \\
\hline \hline \multicolumn{2}{|c|}{} & \multicolumn{4}{c|}{$\%$ of deviations by position } \\
\cline { 3 - 7 } & 2 & 3 & 4 & 5 & 6 \\
\hline Bayesian posterior & $0-20$ & .00 & .00 & .00 & .00 & .00 \\
probabilities that state & $20-40$ & .00 & .00 & .00 & .00 & .00 \\
A would have realized (\%) & $40-60$ & 15.00 & .00 & .00 & .00 & .00 \\
& $60-80$ & .00 & .00 & .00 & .00 & .00 \\
& $80-100$ & .00 & .00 & .00 & 1.79 & .00 \\
\hline
\end{tabular}

Table 21: Percentage of deviations from Bayesian posterior probability for each position and each level of posterior: Overconfidence in seniority 


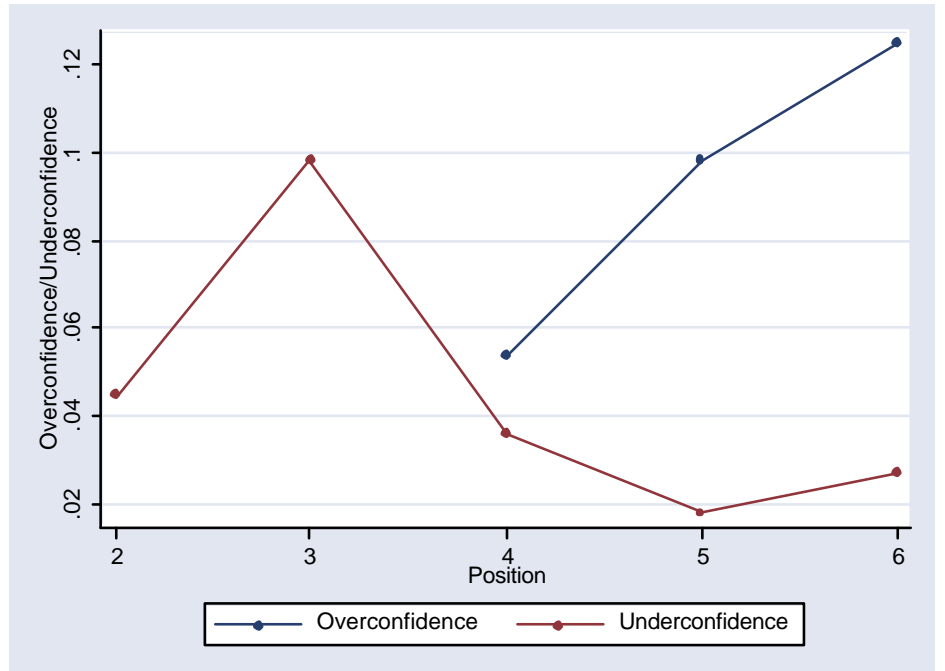

Figure 1: Proportions of overconfidence and underconfidence: Anti-seniority

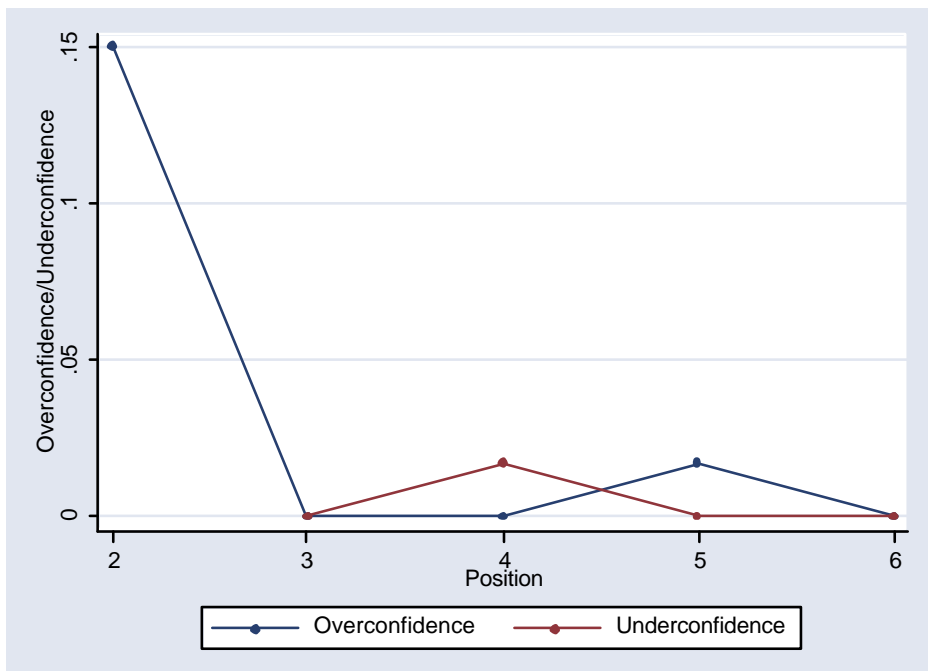

Figure 2: Proportions of overconfidence and underconfidence: Seniority 


\section{B Instructions for subjects}

\section{Instruction}

\section{Welcome!}

This experiment is for the study of economic decision-making. The instructions are simple, and if you follow them carefully and you make good decisions, you can earn a considerable amount of money which will be paid to you in cash after the experiment.

\section{Your task}

At the beginning of each round, the experimenter will draw one of the two cards from covered box. On a card a letter either "A" or "B" is printed. We call the letter printed on the card as the "state" for that round. The experimenter will confirm the letter so as not to be seen by you and hide it until the round finishes. Your task in this experiment is to predict the state for that round.

\section{Order of making prediction}

In this experiment you will make prediction one by one in sequence. Your position of making prediction is determined by drawing one of the six cards. On a card you will draw a number "1", "2", "3", "4", "5", or "6" is printed. This number is your position. For example if you draw the card on which the number "4" is printed, you will make prediction in position 4 .

\section{Information for making prediction}

In predicting the state, you will receive two kinds of information, private signals and predecessors' predictions. You may or may not make use of them.

\section{Private signals}

You can observe private signals by drawing one marble from a covered box. There are twenty white or red marbles in each box. White indicates state "A" and red indicates state "B". The proportion of white and red varies depending on the position, realized state, and treatment and it reflects the preciseness of the signal for predicting the state. The preciseness of the signal is printed below the number of position on the same card you will draw. For example, suppose that under the state "A" you would have drawn card printed "1" and ".55" in Treatment A. Then you draw one marble from a box containing 11 white and 9 red marbles. Thus, the probability that you draw a white marble from the box is $11 / 20=.55$. Therefore the probability 
that your draw indicates the correct state is .55. In another example, suppose that under the state "B" you would have drawn card printed "6" and ".8" in treatment $\mathrm{A}$. Then you draw one marble from a box containing 4 white and 16 red marbles. In this case the probability that your draw indicates the correct state is $16 / 20=.8$.

2 treatments will be conducted in this experiment. In treatment A, you will make predictions in ascending order of the signal preciseness. That is, the subject who has the least precise signal (.55) makes the prediction in position 1 , the subject who has the second least precise signal (.6) makes the prediction in position 2 , and in this manner, the subject who has the most precise signal (.8) makes the prediction in position 6 (last position). In treatment $\mathrm{S}$, you make predictions in descending order of the signal preciseness. That is, the subject who has the most precise signal (.8) makes the prediction in position 1 , the subjects who has the second most precise signal (.75) makes the prediction in position 2 , and in this manner, the subject who has the least precise signal (.55) makes the prediction in position 6 (last position). Which treatment is conducted is announced at the beginning of each round.

The following tables summarize proportions of white and red marbles for each position, realized state, and treatment. Note that your signals indicate the correct state more accurately than random guessing in any case because signal preciseness is always higher than .5 .

\begin{tabular}{|c|c|c|c|c|c|c|c|}
\hline \multicolumn{8}{|c|}{ Preatment A } \\
\hline \hline \multicolumn{2}{|c|}{} & \multicolumn{7}{c|}{ Position (Signal preciseness) } \\
\hline State realized & Marbles & $1(.55)$ & $2(.6)$ & $3(.65)$ & $4(.7)$ & $5(.75)$ & $6(.8)$ \\
A & White & 11 & 12 & 13 & 14 & 15 & 16 \\
& Red & 9 & 8 & 7 & 6 & 5 & 4 \\
& White & 9 & 8 & 7 & 6 & 5 & 4 \\
& Red & 11 & 12 & 13 & 14 & 15 & 16 \\
\hline \hline \multicolumn{8}{|c|}{ Treatment S } \\
\hline \hline \multicolumn{7}{|c|}{ Position (Signal preciseness) } \\
\hline State realized & Marbles & $1(.8)$ & $2(.75)$ & $3(7)$ & $4(.65)$ & $5(.6)$ & $6(.55)$ \\
A & White & 16 & 15 & 14 & 13 & 12 & 11 \\
& Red & 4 & 5 & 6 & 7 & 8 & 9 \\
B & White & 4 & 5 & 6 & 7 & 8 & 9 \\
& Red & 16 & 15 & 14 & 13 & 12 & 11 \\
\hline
\end{tabular}

Predecessors' predictions

In addition to private signals you will observe your predecessors' predictions. Once a subject submits a prediction, the experimenter will record it 
on the experimenter's record sheet and show it to other subjects in later positions. For example, the subject in position 2 can observe the prediction made by the subject in position 1, the subject in position 3 can observe two predictions made by the subjects in positions 1 and 2 , the subject in position 6 (last position) can observe five predictions made by the subjects in positions from 1 to 5 , and so on.

Thus, the subjects assigned to later than position 2 make predictions by referring to both private signals and all of their predecessors' predictions whereas the subject in position 1 can observe only his or her private signal.

\section{Procedure of the experiments}

We call a sequence of making predictions by six subjects a round. In today's experiment you will make predictions for sixteen rounds. In each round, experiment is conducted in the following procedure.

1. The experimenter announces which treatment to be conducted for that round.

2. The experimenter draw a card which determines the state for that round. After confirming the letter the experimenter hide it from you.

3. You draw one card on which the position of making prediction and the signal preciseness are written.

4. The experimenter approaches you one by one from position 1 to position 6 and present one box which contains the exact proportion of white and red marbles for that position, realized state, and treatment.

5. You draw one marble from the box.

6. After confirming the color of the marble, you replace it into the box and write down the state indicated by the color of the marble in the space printed "Your Signal" on the subject's record sheet. At the same time the experimenter also record it on the experimenter's record sheet.

7. 3-6 is repeated until all of you observe the private signals.

8. You make your prediction when the experimenter approaches you and tells you to do so. Before making a prediction, the experimenter shows a sequence of your predecessors' predictions to you. You write down them in the space printed "Your Predecessors' Predictions". A prediction is made by writing down one of the two states you think which is more 
likely to be in the space printed "Your Prediction" on the subject's record sheet. Do not write your prediction until you are told to do so.

9. Once a prediction is made, the experimenter checks it and write it down on the experimenter's record sheet. Then, the experimenter approaches the subject in the next position and show the sequence of his or her predecessors' predictions.

10. 8-9 is repeated until all of you make predictions.

11. The card the experimenter have drawn is revealed. The experimenter record the correct and incorrect predictions for each subject on the experimenter's record sheet.

\section{Payment}

Your payoff for participating this experiment depends on the number of correct predictions. You will be paid 200 yen for a correct prediction and 0 yen for an incorrect prediction. Sum of the results for all 16 predictions will be paid to you privately after the experiment. The theoretical possible maximum payment is 3200 yen and the minimum is 0 yen.

\section{Your information}

Your private information concerning this experiment is not known to anyone. Your name, address, student ID number, predictions, payoffs are confidential.

\section{Notice}

This experiment is conducted based on scientific method. Please note followings.

- Do not talk with others.

- Do not see others' draw and record sheet.

- Do not write your signals and predictions until you are told to do so.

If you do not follow these instructions, you will be dismissed. In this case, you cannot receive payoff you earned until then.

If you have any questions, raise your hand. 


\section{Bayesian posterior probability that state A would have realized at each decision node ${ }^{19}$}

\section{C.1 Anti-seniority}

\begin{tabular}{|c|c|c|c|}
\hline $\operatorname{Pr}\left(A \mid \sigma_{A}^{1}\right)$ & .55 & $\operatorname{Pr}\left(A \mid \pi_{A}^{1}, \pi_{A}^{2}, \pi_{A}^{3}, \pi_{A}^{4}, \sigma_{A}^{5}\right)$ & .959732 \\
\hline $\operatorname{Pr}\left(A \mid \sigma_{B}^{1}\right)$ & 45 & $\operatorname{Pr}\left(A \mid \pi_{A}^{1}, \pi_{A}^{2}, \pi_{A}^{3}, \pi_{A}^{4}, \sigma_{B}^{5}\right)$ & \\
\hline $\operatorname{Pr}\left(A \mid \pi_{A}^{1}, \sigma_{A}^{2}\right)$ & 6470 & $\operatorname{Pr}\left(A \mid \pi_{A}^{1}, \pi_{A}^{2}, \pi_{A}^{3}, \pi_{B}^{4}, \sigma_{A}^{5}\right)$ & 814042 \\
\hline $\mathrm{r}\left(A \mid \pi_{A}^{1}, \sigma_{B}^{2}\right)$ & 448 & $\operatorname{Pr}\left(A \mid \pi_{A}^{1}, \pi_{A}^{2}, \pi_{A}^{3}, \pi\right.$ & 327231 \\
\hline $\mathrm{r}\left(A \mid \pi_{B}^{1}, \sigma_{A}^{2}\right)$ & .551 & $\operatorname{Pr}\left(A \mid \pi_{A}^{1}, \pi_{A}^{2}, \pi_{B}^{3}, \pi\right.$ & 873582 \\
\hline $\mathrm{r}\left(A \mid \pi_{B}^{1}, \sigma_{B}^{2}\right)$ & 352941 & $\operatorname{Pr}\left(A \mid \pi_{A}^{1}, \pi_{A}^{2}, \pi_{B}^{3}, \pi\right.$ & 434327 \\
\hline$r\left(A \mid \pi_{A}^{1}, \pi_{A}^{2}, \sigma_{A}^{3}\right)$ & .772973 & $\operatorname{Pr}\left(A \mid \pi_{A}^{1}, \pi_{B}^{2}, \pi_{A}^{3}, \pi_{A}^{4}, \sigma_{A}^{5}\right)$ & 913738 \\
\hline $\mathrm{r}\left(A \mid \pi_{A}^{1}, \pi_{A}^{2}, \sigma_{B}^{3}\right)$ & .496774 & $\operatorname{Pr}\left(A \mid \pi_{A}^{1}, \pi_{B}^{2}, \pi_{A}^{3}, \pi_{A}^{4}, \sigma_{B}^{5}\right)$ & 540643 \\
\hline $\mathrm{r}\left(A \mid \pi_{A}^{1}, \pi_{B}^{2}, \sigma_{A}^{3}\right)$ & .6021. & $\operatorname{Pr}\left(A \mid \pi_{B}^{1}, \pi_{A}^{2}, \pi_{A}^{3}, \pi_{A}^{4}, \sigma_{A}^{5}\right)$ & 941019 \\
\hline $\mathrm{r}\left(A \mid \pi_{A}^{1}, \pi_{B}^{2}, \sigma_{B}^{3}\right)$ & .30496 & $\operatorname{Pr}\left(A \mid \pi_{B}^{1}, \pi_{A}^{2}, \pi_{A}^{3}, \pi_{A}^{4}, \sigma_{B}^{5}\right)$ & 639344 \\
\hline$\left(A \mid \pi_{B}^{1}, \pi_{A}^{2}, \sigma_{A}^{3}\right)$ & .69505 & $\operatorname{Pr}\left(A \mid \pi_{A}^{1}, \pi_{A}^{2}, \pi_{B}^{3}, \pi_{B}^{4}, \sigma_{A}^{5}\right)$ & 559322 \\
\hline $\mathrm{r}\left(A \mid \pi_{B}^{1}, \pi_{A}^{2}, \sigma_{B}^{3}\right)$ & 397895 & $\operatorname{Pr}\left(A \mid \pi_{A}^{1}, \pi_{A}^{2}, \pi_{B}^{3}, \pi_{B}^{4}, \sigma_{B}^{5}\right)$ & 123596 \\
\hline$\left(A \mid \pi_{B}^{1}, \pi_{B}^{2}, \sigma_{A}^{3}\right)$ & .503226 & $\operatorname{Pr}\left(A \mid \pi_{A}^{1}, \pi_{B}^{2}, \pi_{B}^{3}, ?\right.$ & 374 \\
\hline $\mathrm{r}\left(A \mid \pi_{B}^{1}, \pi_{B}^{2}, \sigma_{B}^{3}\right)$ & 227027 & $\operatorname{Pr}\left(A \mid \pi_{A}^{1}, \pi_{B}^{2}, \pi_{B}^{3}, \pi_{A}^{4}, \sigma_{B}^{5}\right)$ & 254425 \\
\hline $\operatorname{Pr}\left(A \mid \pi_{A}^{1}, \pi_{A}^{2}, \pi_{A}^{3}, \sigma_{A}^{4}\right)$ & .888199 & $\operatorname{Pr}\left(A \mid \pi_{B}^{1}, \pi_{B}^{2}, \pi_{A}^{3}, \pi_{A}^{4}, \sigma_{A}^{5}\right)$ & 876404 \\
\hline $\operatorname{Pr}\left(A \mid \pi_{A}^{1}, \pi_{A}^{2}, \pi_{A}^{3}, \sigma_{B}^{4}\right)$ & .593361 & $\operatorname{Pr}\left(A \mid \pi_{B}^{1}, \pi_{B}^{2}, \pi_{A}^{3}, \pi_{A}^{4}, \sigma_{B}^{5}\right)$ & \\
\hline $\mathrm{r}\left(A \mid \pi_{A}^{1}, \pi_{A}^{2}, \pi_{B}^{3}, \sigma_{A}^{4}\right)$ & .697283 & $\operatorname{Pr}\left(A \mid \pi_{A}^{1}, \pi_{B}^{2}, \pi_{A}^{3}, \pi_{B}^{4}, \sigma_{A}^{5}\right)$ & 660508 \\
\hline $\mathrm{r}\left(A \mid \pi_{A}^{1}, \pi_{A}^{2}, \pi_{B}^{3}, \sigma_{B}^{4}\right)$ & .297297 & $\operatorname{Pr}\left(A \mid \pi_{A}^{1}, \pi_{B}^{2}, \pi_{A}^{3}, \pi_{B}^{4}, \sigma_{B}^{5}\right)$ & \\
\hline $\mathrm{r}\left(A \mid \pi_{A}^{1}, \pi_{B}^{2}, \pi_{A}^{3}, \sigma_{A}^{4}\right)$ & .779292 & $\operatorname{Pr}\left(A \mid \pi_{B}^{1}, \pi_{A}^{2}, \pi_{B}^{3}, \pi_{A}^{4}, \sigma_{A}^{5}\right)$ & 82225 \\
\hline $\mathrm{r}\left(A \mid \pi_{A}^{1}, \pi_{B}^{2}, \pi_{A}^{3}, \sigma_{B}^{4}\right)$ & .3933 & $\operatorname{Pr}\left(A \mid \pi_{B}^{1}, \pi_{A}^{2}, \pi_{B}^{3}, \pi_{A}^{4}, \sigma_{B}^{5}\right)$ & 492 \\
\hline$r\left(A \mid \pi_{B}^{1}, \pi_{A}^{2}, \pi_{A}^{3}, \sigma_{A}^{4}\right)$ & .841727 & $\operatorname{Pr}\left(A \mid \pi_{B}^{1}, \pi_{A}^{2}, \pi_{A}^{3}, \pi_{B}^{4}, \sigma_{A}^{5}\right)$ & 745575 \\
\hline $\mathrm{r}\left(A \mid \pi_{B}^{1}, \pi_{A}^{2}, \pi_{A}^{3}, \sigma_{B}^{4}\right)$ & .4941 & $\operatorname{Pr}\left(A \mid \pi_{B}^{1}, \pi_{A}^{2}, \pi_{A}^{3}, \pi_{B}^{4}, \sigma_{B}^{5}\right)$ & 245626 \\
\hline $\mathrm{r}\left(A \mid \pi_{A}^{1}, \pi_{B}^{2}, \pi_{B}^{3}, \sigma_{A}^{4}\right)$ & .505866 & $\operatorname{Pr}\left(A \mid \pi_{A}^{1}, \pi_{B}^{2}, \pi_{B}^{3}, \pi_{B}^{4}, \sigma_{A}^{5}\right)$ & 360656 \\
\hline $\mathrm{r}\left(A \mid \pi_{A}^{1}, \pi_{B}^{2}, \pi_{B}^{3}, \sigma_{B}^{4}\right)$ & 158273 & $\operatorname{Pr}\left(A \mid \pi_{A}^{1}, \pi_{B}^{2}, \pi_{B}^{3}, \pi_{B}^{4}, \sigma_{B}^{5}\right)$ & .058981 \\
\hline $\operatorname{Pr}\left(A \mid \pi_{B}^{1}, \pi_{B}^{2}, \pi_{A}^{3}, \sigma_{A}^{4}\right)$ & .702703 & $\operatorname{Pr}\left(A \mid \pi_{B}^{1}, \pi_{A}^{2}, \pi_{B}^{3}, \pi_{B}^{4}, \sigma_{A}^{5}\right)$ & .459357 \\
\hline $\operatorname{Pr}\left(A \mid \pi_{B}^{1}, \pi_{B}^{2}, \pi_{A}^{3}, \sigma_{B}^{4}\right)$ & .3027 & $\operatorname{Pr}\left(A \mid \pi_{B}^{1}, \pi_{A}^{2}, \pi_{B}^{3}, \pi_{B}^{4}, \sigma_{B}^{5}\right)$ & .080262 \\
\hline $\operatorname{Pr}\left(A \mid \pi_{B}^{1}, \pi_{A}^{2}, \pi_{B}^{3}, \sigma_{A}^{4}\right)$ & 606602 & $\operatorname{Pr}\left(A \mid \pi_{B}^{1}, \pi_{B}^{2}, \pi_{A}^{3}, \pi_{B}^{4}, \sigma_{A}^{5}\right)$ & .565673 \\
\hline $\mathrm{r}\left(A \mid \pi_{B}^{1}, \pi_{A}^{2}, \pi_{B}^{3}, \sigma_{B}^{4}\right)$ & .220708 & $\operatorname{Pr}\left(A \mid \pi_{B}^{1}, \pi_{B}^{2}, \pi_{A}^{3}, \pi_{B}^{4}, \sigma_{B}^{5}\right)$ & .126418 \\
\hline $\operatorname{Pr}\left(A \mid \pi_{B}^{1}, \pi_{B}^{2}, \pi_{B}^{3}, \sigma_{A}^{4}\right)$ & .406639 & $\operatorname{Pr}\left(A \mid \pi_{B}^{1}, \pi_{B}^{2}, \pi_{B}^{3}, \pi_{A}^{4}, \sigma_{A}^{5}\right)$ & .672769 \\
\hline $\operatorname{Pr}\left(A \mid \pi_{B}^{1}, \pi_{B}^{2}, \pi_{B}^{3}, \sigma_{B}^{4}\right)$ & .111801 & $\operatorname{Pr}\left(A \mid \pi_{B}^{1}, \pi_{B}^{2}, \pi_{B}^{3}, \pi_{A}^{4}, \sigma_{B}^{5}\right)$ & .185958 \\
\hline
\end{tabular}

\footnotetext{
${ }^{19}$ Bayesian posteriors are typed in italics if imbalance between Bayesian posterior and private signal occurs.
} 


\begin{tabular}{|c|c|c|c|}
\hline $\operatorname{Pr}\left(A \mid \pi_{B}^{1}, \pi_{B}^{2}, \pi_{B}^{3}, \pi_{B}^{4}, \sigma_{A}^{5}\right)$ & 274112 & $\operatorname{Pr}\left(A \mid \pi_{B}^{1}, \pi_{A}^{2}, \pi_{A}^{3}, \pi_{B}^{4}, \pi_{A}^{5}, \sigma_{A}^{6}\right)$ & .921 \\
\hline $\operatorname{Pr}\left(A \mid \pi_{B}^{1}, \pi_{B}^{2}, \pi_{B}^{3}, \pi_{B}^{4}, \sigma_{B}^{5}\right)$ & 040268 & $\operatorname{Pr}\left(A \mid \pi_{B}^{1}, \pi_{A}^{2}, \pi_{A}^{3}, \pi_{B}^{4}, \pi_{A}^{5}, \sigma_{B}^{6}\right)$ & 422835 \\
\hline $\operatorname{Pr}\left(A \mid \pi_{A}^{1}, \pi_{A}^{2}, \pi_{A}^{3}, \pi_{A}^{4}, \pi_{A}^{5}, \sigma_{A}^{6}\right)$ & 989619 & $\operatorname{Pr}\left(A \mid \pi_{B}^{1}, \pi_{A}^{2}, \pi_{B}^{3}, \pi_{A}^{4}, \pi_{A}^{5}, \sigma_{A}^{6}\right)$ & 948727 \\
\hline $\operatorname{Pr}\left(A \mid \pi_{A}^{1}, \pi_{A}^{2}, \pi_{A}^{3}, \pi_{A}^{4}, \pi_{A}^{5}, \sigma_{B}^{6}\right)$ & 856287 & $\operatorname{Pr}\left(A \mid \pi_{B}^{1}, \pi_{A}^{2}, \pi_{B}^{3}, \pi_{A}^{4}, \pi_{A}^{5}, \sigma_{B}^{6}\right)$ & .536279 \\
\hline $\operatorname{Pr}\left(A \mid \pi_{A}^{1}, \pi_{A}^{2}, \pi_{A}^{3}, \pi_{A}^{4}, \pi_{B}^{5}, \sigma_{A}^{6}\right)$ & 913738 & $\operatorname{Pr}\left(A \mid \pi_{A}^{1}, \pi_{A}^{2}, \pi_{B}^{3}, \pi_{B}^{4}, \pi_{B}^{5}, \sigma_{A}^{6}\right)$ & 360656 \\
\hline $\operatorname{Pr}\left(A \mid \pi_{A}^{1}, \pi_{A}^{2}, \pi_{A}^{3}, \pi_{A}^{4}, \pi_{B}^{5}, \sigma_{B}^{6}\right)$ & 398329 & $\operatorname{Pr}\left(A \mid \pi_{A}^{1}, \pi_{A}^{2}, \pi_{B}^{3}, \pi_{B}^{4}, \pi_{B}^{5}, \sigma_{B}^{6}\right)$ & 56 \\
\hline $\operatorname{Pr}\left(A \mid \pi_{A}^{1}, \pi_{A}^{2}, \pi_{A}^{3}, \pi_{B}^{4}, \pi_{A}^{5}, \sigma_{A}^{6}\right)$ & .945976 & $\operatorname{Pr}\left(A \mid \pi_{A}^{1}, \pi_{B}^{2}, \pi_{A}^{3}, \pi_{B}^{4}, \pi_{B}^{5}, \sigma_{A}^{6}\right)$ & 721 \\
\hline $\operatorname{Pr}\left(A \mid \pi_{A}^{1}, \pi_{A}^{2}, \pi_{A}^{3}, \pi_{B}^{4}, \pi_{A}^{5}, \sigma_{B}^{6}\right)$ & .522533 & $\operatorname{Pr}\left(A \mid \pi_{A}^{1}, \pi_{B}^{2}, \pi_{A}^{3}, \pi_{B}^{4}, \pi_{B}^{5}, \sigma_{B}^{6}\right)$ & .051273 \\
\hline $\operatorname{Pr}\left(A \mid \pi_{A}^{1}, \pi_{A}^{2}, \pi_{B}^{3}, \pi_{A}^{4}, \pi_{A}^{5}, \sigma_{A}^{6}\right)$ & .965085 & $\operatorname{Pr}\left(A \mid \pi_{A}^{1}, \pi_{B}^{2}, \pi_{B}^{3}, \pi_{A}^{4}, \pi_{B}^{5}, \sigma_{A}^{6}\right)$ & .577 \\
\hline $\operatorname{Pr}\left(A \mid \pi_{A}^{1}, \pi_{A}^{2}, \pi_{B}^{3}, \pi_{A}^{4}, \pi_{A}^{5}, \sigma_{B}^{6}\right)$ & .633373 & $\operatorname{Pr}\left(A \mid \pi_{A}^{1}, \pi_{B}^{2}, \pi_{B}^{3}, \pi_{A}^{4}, \pi_{B}^{5}, \sigma_{B}^{6}\right)$ & 606 \\
\hline $\operatorname{Pr}\left(A \mid \pi_{A}^{1}, \pi_{B}^{2}, \pi_{A}^{3}, \pi_{A}^{4}, \pi_{A}^{5}, \sigma_{A}^{6}\right)$ & .976943 & $\operatorname{Pr}\left(A \mid \pi_{A}^{1}, \pi_{B}^{2}, \pi_{B}^{3}, \pi_{B}^{4}, \pi_{A}^{5}, \sigma_{A}^{6}\right)$ & 692913 \\
\hline $\operatorname{Pr}\left(A \mid \pi_{A}^{1}, \pi_{B}^{2}, \pi_{A}^{3}, \pi_{A}^{4}, \pi_{A}^{5}, \sigma_{B}^{6}\right)$ & 725888 & $\operatorname{Pr}\left(A \mid \pi_{A}^{1}, \pi_{B}^{2}, \pi_{B}^{3}, \pi_{B}^{4}, \pi_{A}^{5}, \sigma_{B}^{6}\right)$ & \\
\hline $\operatorname{Pr}\left(A \mid \pi_{B}^{1}, \pi_{A}^{2}, \pi_{A}^{3}, \pi_{A}^{4}, \pi_{A}^{5}, \sigma_{A}^{6}\right)$ & 984572 & $\operatorname{Pr}\left(A \mid \pi_{B}^{1}, \pi_{A}^{2}, \pi_{A}^{3}, \pi_{B}^{4}, \pi_{B}^{5}, \sigma_{A}^{6}\right)$ & .565673 \\
\hline $\operatorname{Pr}\left(A \mid \pi_{B}^{1}, \pi_{A}^{2}, \pi_{A}^{3}, \pi_{A}^{4}, \pi_{A}^{5}, \sigma_{B}^{6}\right)$ & .799544 & $\operatorname{Pr}\left(A \mid \pi_{B}^{1}, \pi_{A}^{2}, \pi_{A}^{3}, \pi_{B}^{4}, \pi_{B}^{5}, \sigma_{B}^{6}\right)$ & 273 \\
\hline $\operatorname{Pr}\left(A \mid \pi_{A}^{1}, \pi_{A}^{2}, \pi_{A}^{3}, \pi_{B}^{4}, \pi_{B}^{5}, \sigma_{A}^{6}\right)$ & .660508 & $\operatorname{Pr}\left(A \mid \pi_{B}^{1}, \pi_{A}^{2}, \pi_{B}^{3}, \pi_{A}^{4}, \pi_{B}^{5}, \sigma_{A}^{6}\right)$ & 672769 \\
\hline $\operatorname{Pr}\left(A \mid \pi_{A}^{1}, \pi_{A}^{2}, \pi_{A}^{3}, \pi_{B}^{4}, \pi_{B}^{5}, \sigma_{B}^{6}\right)$ & 108415 & $\operatorname{Pr}\left(A \mid \pi_{B}^{1}, \pi_{A}^{2}, \pi_{B}^{3}, \pi_{A}^{4}, \pi_{B}^{5}, \sigma_{B}^{6}\right)$ & 65 \\
\hline $\mathrm{r}\left(A \mid \pi_{A}^{1}, \pi_{A}^{2}, \pi_{B}^{3}, \pi_{B}^{4}, \pi_{A}^{5}, \sigma_{A}^{6}\right)$ & 835443 & $\operatorname{Pr}\left(A \mid \pi_{B}^{1}, \pi_{A}^{2}, \pi_{B}^{3}, \pi_{B}^{4}, \pi_{A}^{5}, \sigma_{A}^{6}\right)$ & .772 \\
\hline $\mathrm{r}\left(A \mid \pi_{A}^{1}, \pi_{A}^{2}, \pi_{B}^{3}, \pi_{B}^{4}, \pi_{A}^{5}, \sigma_{B}^{6}\right)$ & .240876 & $\operatorname{Pr}\left(A \mid \pi_{B}^{1}, \pi_{A}^{2}, \pi_{B}^{3}, \pi_{B}^{4}, \pi_{A}^{5}, \sigma_{B}^{6}\right)$ & 98 \\
\hline $\mathrm{r}\left(A \mid \pi_{A}^{1}, \pi_{B}^{2}, \pi_{B}^{3}, \pi_{A}^{4}, \pi_{A}^{5}, \sigma_{A}^{6}\right)$ & .924727 & $\operatorname{Pr}\left(A \mid \pi_{B}^{1}, \pi_{B}^{2}, \pi_{A}^{3}, \pi_{A}^{4}, \pi_{B}^{5}, \sigma_{A}^{6}\right)$ & \\
\hline $\operatorname{Pr}\left(A \mid \pi_{A}^{1}, \pi_{B}^{2}, \pi_{B}^{3}, \pi_{A}^{4}, \pi_{A}^{5}, \sigma_{B}^{6}\right)$ & .434327 & $\operatorname{Pr}\left(A \mid \pi_{B}^{1}, \pi_{B}^{2}, \pi_{A}^{3}, \pi_{A}^{4}, \pi_{B}^{5}, \sigma_{B}^{6}\right)$ & 164557 \\
\hline $\operatorname{Pr}\left(A \mid \pi_{B}^{1}, \pi_{B}^{2}, \pi_{A}^{3}, \pi_{A}^{4}, \pi_{A}^{5}, \sigma_{A}^{6}\right)$ & .965944 & $\operatorname{Pr}\left(A \mid \pi_{B}^{1}, \pi_{B}^{2}, \pi_{A}^{3}, \pi_{B}^{4}, \pi_{A}^{5}, \sigma_{A}^{6}\right)$ & \\
\hline $\mathrm{r}\left(A \mid \pi_{B}^{1}, \pi_{B}^{2}, \pi_{A}^{3}, \pi_{A}^{4}, \pi_{A}^{5}, \sigma_{B}^{6}\right)$ & .639344 & $\operatorname{Pr}\left(A \mid \pi_{B}^{1}, \pi_{B}^{2}, \pi_{A}^{3}, \pi_{B}^{4}, \pi_{A}^{5}, \sigma_{B}^{6}\right)$ & 245626 \\
\hline $\mathrm{r}\left(A \mid \pi_{A}^{1}, \pi_{A}^{2}, \pi_{B}^{3}, \pi_{A}^{4}, \pi_{B}^{5}, \sigma_{A}^{6}\right)$ & .754374 & $\operatorname{Pr}\left(A \mid \pi_{B}^{1}, \pi_{B}^{2}, \pi_{B}^{3}, \pi\right.$ & 891 \\
\hline $\operatorname{Pr}\left(A \mid \pi_{A}^{1}, \pi_{A}^{2}, \pi_{B}^{3}, \pi_{A}^{4}, \pi_{B}^{5}, \sigma_{B}^{6}\right)$ & .16104 & $\operatorname{Pr}\left(A \mid \pi_{B}^{1}, \pi_{B}^{2}, \pi_{B}^{3}, \pi_{A}^{4}, \pi_{A}^{5}, \sigma_{B}^{6}\right)$ & .339492 \\
\hline $\operatorname{Pr}\left(A \mid \pi_{A}^{1}, \pi_{B}^{2}, \pi_{A}^{3}, \pi_{B}^{4}, \pi_{A}^{5}, \sigma_{A}^{6}\right)$ & .886135 & $\operatorname{Pr}\left(A \mid \pi_{A}^{1}, \pi_{B}^{2}, \pi_{B}^{3}, \pi_{B}^{4}, \pi_{B}^{5}, \sigma_{A}^{6}\right)$ & .200456 \\
\hline $\operatorname{Pr}\left(A \mid \pi_{A}^{1}, \pi_{B}^{2}, \pi_{A}^{3}, \pi_{B}^{4}, \pi_{A}^{5}, \sigma_{B}^{6}\right)$ & .327231 & $\operatorname{Pr}\left(A \mid \pi_{A}^{1}, \pi_{B}^{2}, \pi_{B}^{3}, \pi_{B}^{4}, \pi_{B}^{5}, \sigma_{B}^{6}\right)$ & .015 \\
\hline $\operatorname{Pr}\left(A \mid \pi_{A}^{1}, \pi_{B}^{2}, \pi_{A}^{3}, \pi_{A}^{4}, \pi_{B}^{5}, \sigma_{A}^{6}\right)$ & .824802 & $\operatorname{Pr}\left(A \mid \pi_{B}^{1}, \pi_{A}^{2}, \pi_{B}^{3}, \pi_{B}^{4}, \pi_{B}^{5}, \sigma_{A}^{6}\right)$ & .274112 \\
\hline $\operatorname{Pr}\left(A \mid \pi_{A}^{1}, \pi_{B}^{2}, \pi_{A}^{3}, \pi_{A}^{4}, \pi_{B}^{5}, \sigma_{B}^{6}\right)$ & .227345 & $\operatorname{Pr}\left(A \mid \pi_{B}^{1}, \pi_{A}^{2}, \pi_{B}^{3}, \pi_{B}^{4}, \pi_{B}^{5}, \sigma_{B}^{6}\right)$ & .023057 \\
\hline $\operatorname{Pr}\left(A \mid \pi_{B}^{1}, \pi_{A}^{2}, \pi_{A}^{3}, \pi_{A}^{4}, \pi_{B}^{5}, \sigma_{A}^{6}\right)$ & .876404 & $\operatorname{Pr}\left(A \mid \pi_{B}^{1}, \pi_{B}^{2}, \pi_{A}^{3}, \pi_{B}^{4}, \pi_{B}^{5}, \sigma_{A}^{6}\right)$ & $.36662^{7}$ \\
\hline $\operatorname{Pr}\left(A \mid \pi_{B}^{1}, \pi_{A}^{2}, \pi_{A}^{3}, \pi_{A}^{4}, \pi_{B}^{5}, \sigma_{B}^{6}\right)$ & .307087 & $\operatorname{Pr}\left(A \mid \pi_{B}^{1}, \pi_{B}^{2}, \pi_{A}^{3}, \pi_{B}^{4}, \pi_{B}^{5}, \sigma_{B}^{6}\right)$ & .034915 \\
\hline
\end{tabular}

\begin{tabular}{|c|c|}
\hline $\operatorname{Pr}\left(A \mid \pi_{B}^{1}, \pi_{B}^{2}, \pi_{B}^{3}, \pi_{A}^{4}, \pi_{B}^{5}, \sigma_{A}^{6}\right)$ & .477467 \\
\hline $\operatorname{Pr}\left(A \mid \pi_{B}^{1}, \pi_{B}^{2}, \pi_{B}^{3}, \pi_{A}^{4}, \pi_{B}^{5}, \sigma_{B}^{6}\right)$ & .054024 \\
\hline $\operatorname{Pr}\left(A \mid \pi_{B}^{1}, \pi_{B}^{2}, \pi_{B}^{3}, \pi_{B}^{4}, \pi_{A}^{5}, \sigma_{A}^{6}\right)$ & .601671 \\
\hline $\operatorname{Pr}\left(A \mid \pi_{B}^{1}, \pi_{B}^{2}, \pi_{B}^{3}, \pi_{B}^{4}, \pi_{A}^{5}, \sigma_{B}^{6}\right)$ & .086262 \\
\hline $\operatorname{Pr}\left(A \mid \pi_{B}^{1}, \pi_{B}^{2}, \pi_{B}^{3}, \pi_{B}^{4}, \pi_{B}^{5}, \sigma_{A}^{6}\right)$ & .143713 \\
\hline $\operatorname{Pr}\left(A \mid \pi_{B}^{1}, \pi_{B}^{2}, \pi_{B}^{3}, \pi_{B}^{4}, \pi_{B}^{5}, \sigma_{B}^{6}\right)$ & .010381 \\
\hline
\end{tabular}




\section{C.2 Seniority}

\begin{tabular}{|c|c|c|c|}
\hline $\operatorname{Pr}\left(A \mid \sigma_{A}^{1}\right)$ & 8 & $\operatorname{Pr}\left(A \mid \pi_{A}^{1}, \pi_{A}^{2}, \pi_{A}^{3}, \pi_{A}^{4}, \sigma_{A}^{5}\right)$ & \\
\hline $\operatorname{Pr}\left(A \mid \sigma_{B}^{1}\right)$ & 2 & $\operatorname{Pr}\left(A \mid \pi_{A}^{1}, \pi_{A}^{2}, \pi_{A}^{3}, \pi_{A}^{4}, \sigma_{B}^{5}\right)$ & 971963 \\
\hline$\left(A \mid \pi_{A}^{1}, \sigma_{A}^{2}\right)$ & 923( & $\operatorname{Pr}\left(A \mid \pi_{A}^{1}, \pi_{A}^{2}, \pi_{A}^{3}, \pi_{B}^{4}, \sigma_{A}^{5}\right)$ & \\
\hline $\mathrm{r}\left(A \mid \pi_{A}^{1}, \sigma_{B}^{2}\right)$ & 571729 & $\operatorname{Pr}\left(A \mid \pi_{A}^{1}, \pi_{A}^{2}, \pi_{A}^{3}, \pi_{B}^{4}, \sigma_{B}^{5}\right)$ & 909513 \\
\hline $\operatorname{Pr}\left(A \mid \pi_{B}^{1}, \sigma_{A}^{2}\right)$ & .428571 & $\operatorname{Pr}\left(A \mid \pi_{A}^{1}, \pi_{A}^{2}, \pi_{B}^{3}, \pi\right.$ & 754 \\
\hline $\mathrm{r}\left(A \mid \pi_{B}^{1}, \sigma_{B}^{2}\right)$ & .076923 & $\operatorname{Pr}\left(A \mid \pi_{A}^{1}, \pi_{A}^{2}, \pi_{B}^{3}, \pi_{A}^{4}\right.$, & 864266 \\
\hline $\mathrm{r}\left(A \mid \pi_{A}^{1}, \pi_{A}^{2}, \sigma_{A}^{3}\right)$ & .965 & $\operatorname{Pr}\left(A \mid \pi_{A}^{1}, \pi_{B}^{2}, \pi_{A}^{3}, \tau\right.$ & 896552 \\
\hline $\operatorname{Pr}\left(A \mid \pi_{A}^{1}, \pi_{A}^{2}, \sigma_{B}^{3}\right)$ & .837209 & $\operatorname{Pr}\left(A \mid \pi_{A}^{1}, \pi_{B}^{2}, \pi_{A}^{3}, \pi_{A}^{4}, \sigma_{B}^{5}\right)$ & 793893 \\
\hline $\operatorname{Pr}\left(A \mid \pi_{A}^{1}, \pi_{B}^{2}, \sigma_{A}^{3}\right)$ & .756 & $\operatorname{Pr}\left(A \mid \pi_{B}^{1}, \pi_{A}^{2}, \pi_{A}^{3}, \pi\right.$ & 829787 \\
\hline $\operatorname{Pr}\left(A \mid \pi_{A}^{1}, \pi_{B}^{2}, \sigma_{B}^{3}\right)$ & .363636 & $\operatorname{Pr}\left(A \mid \pi_{B}^{1}, \pi_{A}^{2}, \pi_{A}^{3}, \pi_{A}^{4}, \sigma_{B}^{5}\right)$ & 684211 \\
\hline $\operatorname{Pr}\left(A \mid \pi_{B}^{1}, \pi_{A}^{2}, \sigma_{A}^{3}\right)$ & 636364 & $\operatorname{Pr}\left(A \mid \pi_{A}^{1}, \pi_{A}^{2}, \pi_{B}^{3}, \pi_{B}^{4}, \sigma_{A}^{5}\right)$ & 80597 \\
\hline $\operatorname{Pr}\left(A \mid \pi_{B}^{1}, \pi_{A}^{2}, \sigma_{B}^{3}\right)$ & 2432 & $\operatorname{Pr}\left(A \mid \pi_{A}^{1}, \pi_{A}^{2}, \pi_{B}^{3}, \pi_{B}^{4}, \sigma_{B}^{5}\right)$ & .648649 \\
\hline $\mathrm{r}\left(A \mid \pi_{B}^{1}, \pi_{B}^{2}, \sigma_{A}^{3}\right)$ & 162791 & $\operatorname{Pr}\left(A \mid \pi_{A}^{1}, \pi_{B}^{2}, \pi_{B}^{3}, \pi_{A}^{4}, \sigma_{A}^{5}\right)$ & 614173 \\
\hline $\mathrm{r}\left(A \mid \pi_{B}^{1}, \pi_{B}^{2}, \sigma_{B}^{3}\right)$ & .034 & $\operatorname{Pr}\left(A \mid \pi_{A}^{1}, \pi_{B}^{2}, \pi_{B}^{3}, \pi_{A}^{4}, \sigma_{B}^{5}\right)$ & 414343 \\
\hline $\operatorname{Pr}\left(A \mid \pi_{A}^{1}, \pi_{A}^{2}, \pi_{A}^{3}, \sigma_{A}^{4}\right)$ & .981 & $\operatorname{Pr}\left(A \mid \pi_{B}^{1}, \pi_{B}^{2}, \pi_{A}^{3}, \pi_{A}^{4}, \sigma_{A}^{5}\right)$ & 351351 \\
\hline $\operatorname{Pr}\left(A \mid \pi_{A}^{1}, \pi_{A}^{2}, \pi_{A}^{3}, \sigma_{B}^{4}\right)$ & $.93^{\prime}$ & $\operatorname{Pr}\left(A \mid \pi_{B}^{1}, \pi_{B}^{2}, \pi_{A}^{3}, \pi_{A}^{4}, \sigma_{B}^{5}\right)$ & \\
\hline $\mathrm{r}\left(A \mid \pi_{A}^{1}, \pi_{A}^{2}, \pi_{B}^{3}, \sigma_{A}^{4}\right)$ & .9052 & $\operatorname{Pr}\left(A \mid \pi_{A}^{1}, \pi_{B}^{2}, \pi_{A}^{3}, \pi_{B}^{4}, \sigma_{A}^{5}\right)$ & 715328 \\
\hline $\mathrm{r}\left(A \mid \pi_{A}^{1}, \pi_{A}^{2}, \pi_{B}^{3}, \sigma_{B}^{4}\right)$ & .734694 & $\mathrm{r}\left(A \mid \pi_{A}^{1}, \pi_{B}^{2}, \pi_{A}^{3}, \pi_{B}^{4}, \sigma_{B}^{5}\right)$ & .527591 \\
\hline $\mathrm{r}\left(A \mid \pi_{A}^{1}, \pi_{B}^{2}, \pi_{A}^{3}, \sigma_{A}^{4}\right)$ & .852459 & $\operatorname{Pr}\left(A \mid \pi_{B}^{1}, \pi_{A}^{2}, \pi_{B}^{3}, \pi_{A}^{4}, \sigma_{A}^{5}\right)$ & .472409 \\
\hline $\mathrm{r}\left(A \mid \pi_{A}^{1}, \pi_{B}^{2}, \pi_{A}^{3}, \sigma_{B}^{4}\right)$ & .626 & $\operatorname{Pr}\left(A \mid \pi_{B}^{1}, \pi_{A}^{2}, \pi_{B}^{3}, \pi_{A}^{4}, \sigma_{B}^{5}\right)$ & 284672 \\
\hline$\left(A \mid \pi_{B}^{1}, \pi_{A}^{2}, \pi_{A}^{3}, \sigma_{A}^{4}\right)$ & $.764^{\prime}$ & $\operatorname{Pr}\left(A \mid \pi_{B}^{1}, \pi_{A}^{2}, \pi_{A}^{3}, \pi_{B}^{4}, \sigma_{A}^{5}\right)$ & 585657 \\
\hline$r\left(A \mid \pi_{B}^{1}, \pi_{A}^{2}, \pi_{A}^{3}, \sigma_{B}^{4}\right)$ & & $\operatorname{Pr}\left(A \mid \pi_{B}^{1}, \pi_{A}^{2}, \pi_{A}^{3}, \pi_{B}^{4}, \sigma_{B}^{5}\right)$ & 27 \\
\hline $\mathrm{r}\left(A \mid \pi_{A}^{1}, \pi_{B}^{2}, \pi_{B}^{3}, \sigma_{A}^{4}\right)$ & .514 & $\operatorname{Pr}\left(A \mid \pi^{1}, \pi^{2} \quad \pi^{3} \pi^{4} \sigma^{5}\right)$ & 315789 \\
\hline $\mathrm{r}\left(A \mid \pi_{A}^{1}, \pi_{B}^{2}, \pi_{B}^{3}, \sigma_{B}^{4}\right)$ & .235 & $\operatorname{Pr}\left(A \mid \pi_{A}^{1}, \pi_{B}^{2}, \pi_{B}^{3}, \pi_{B}^{4}, \sigma_{B}^{5}\right)$ & 170213 \\
\hline $\mathrm{r}\left(A \mid \pi_{B}^{1}, \pi_{B}^{2}, \pi_{A}^{3}, \sigma_{A}^{4}\right)$ & .265306 & $\operatorname{Pr}\left(A \mid \pi_{B}^{1}, \pi_{A}^{2}, \pi_{B}^{3}, \pi_{B}^{4}, \sigma_{A}^{5}\right)$ & $.20610^{r y}$ \\
\hline $\mathrm{r}\left(A \mid \pi_{B}^{1}, \pi_{B}^{2}, \pi_{A}^{3}, \sigma_{B}^{4}\right)$ & .094 & $\operatorname{Pr}\left(A \mid \pi_{B}^{1}, \pi_{A}^{2}, \pi_{B}^{3}, \pi_{B}^{4}, \sigma_{B}^{5}\right)$ & .103448 \\
\hline $\operatorname{Pr}\left(A \mid \pi_{B}^{1}, \pi_{A}^{2}, \pi_{B}^{3}, \sigma_{A}^{4}\right)$ & .373 & $\operatorname{Pr}\left(A \mid \pi_{B}^{1}, \pi_{B}^{2}, \pi_{A}^{3}, \pi_{B}^{4}, \sigma_{A}^{5}\right)$ & .135734 \\
\hline $\operatorname{Pr}\left(A \mid \pi_{B}^{1}, \pi_{A}^{2}, \pi_{B}^{3}, \sigma_{B}^{4}\right)$ & .147 & $\operatorname{Pr}\left(A \mid \pi_{B}^{1}, \pi_{B}^{2}, \pi_{A}^{3}, \pi_{B}^{4}, \sigma_{B}^{5}\right)$ & .065246 \\
\hline $\operatorname{Pr}\left(A \mid \pi_{B}^{1}, \pi_{B}^{2}, \pi_{B}^{3}, \sigma_{A}^{4}\right)$ & .062201 & $\operatorname{Pr}\left(A \mid \pi_{B}^{1}, \pi_{B}^{2}, \pi_{B}^{3}, \pi_{A}^{4}, \sigma_{A}^{5}\right)$ & .090487 \\
\hline $\operatorname{Pr}\left(A \mid \pi_{B}^{1}, \pi_{B}^{2}, \pi_{B}^{3}, \sigma_{B}^{4}\right)$ & .018868 & $\operatorname{Pr}\left(A \mid \pi_{B}^{1}, \pi_{B}^{2}, \pi_{B}^{3}, \pi_{A}^{4}, \sigma_{B}^{5}\right)$ & .042345 \\
\hline
\end{tabular}




\begin{tabular}{|c|c|c|c|}
\hline $\operatorname{Pr}\left(A \mid \pi_{B}^{1}, \pi_{B}^{2}, \pi_{B}^{3}, \pi_{B}^{4}, \sigma_{A}^{5}\right)$ & 037 & $\operatorname{Pr}\left(A \mid \pi_{B}^{1}, \pi_{A}^{2}, \pi_{A}^{3}, \pi_{B}^{4}, \pi_{A}^{5}, \sigma_{A}^{6}\right)$ & \\
\hline $\operatorname{Pr}\left(A \mid \pi_{B}^{1}, \pi_{B}^{2}, \pi_{B}^{3}, \pi_{B}^{4}, \sigma_{B}^{5}\right)$ & 012658 & $\operatorname{Pr}\left(A \mid \pi_{B}^{1}, \pi_{A}^{2}, \pi_{A}^{3}, \pi_{B}^{4}, \pi_{A}^{5}, \sigma_{B}^{6}\right)$ & .536279 \\
\hline $\operatorname{Pr}\left(A \mid \pi_{A}^{1}, \pi_{A}^{2}, \pi_{A}^{3}, \pi_{A}^{4}, \pi_{A}^{5}, \sigma_{A}^{6}\right)$ & 989619 & $\operatorname{Pr}\left(A \mid \pi_{B}^{1}, \pi_{A}^{2}, \pi_{B}^{3}, \pi_{A}^{4}, \pi_{A}^{5}, \sigma_{A}^{6}\right)$ & .522533 \\
\hline $\operatorname{Pr}\left(A \mid \pi_{A}^{1}, \pi_{A}^{2}, \pi_{A}^{3}, \pi_{A}^{4}, \pi_{A}^{5}, \sigma_{B}^{6}\right)$ & .984572 & $\operatorname{Pr}\left(A \mid \pi_{B}^{1}, \pi_{A}^{2}, \pi_{B}^{3}, \pi_{A}^{4}, \pi_{A}^{5}, \sigma_{B}^{6}\right)$ & 422835 \\
\hline $\operatorname{Pr}\left(A \mid \pi_{A}^{1}, \pi_{A}^{2}, \pi_{A}^{3}, \pi_{A}^{4}, \pi_{B}^{5}, \sigma_{A}^{6}\right)$ & .976943 & $\operatorname{Pr}\left(A \mid \pi_{A}^{1}, \pi_{A}^{2}, \pi_{B}^{3}, \pi_{B}^{4}, \pi_{B}^{5}, \sigma_{A}^{6}\right)$ & 692913 \\
\hline $\operatorname{Pr}\left(A \mid \pi_{A}^{1}, \pi_{A}^{2}, \pi_{A}^{3}, \pi_{A}^{4}, \pi_{B}^{5}, \sigma_{B}^{6}\right)$ & .965944 & $\operatorname{Pr}\left(A \mid \pi_{A}^{1}, \pi_{A}^{2}, \pi_{B}^{3}, \pi_{B}^{4}, \pi_{B}^{5}, \sigma_{B}^{6}\right)$ & $.6016^{r} 1$ \\
\hline $\operatorname{Pr}\left(A \mid \pi_{A}^{1}, \pi_{A}^{2}, \pi_{A}^{3}, \pi_{B}^{4}, \pi_{A}^{5}, \sigma_{A}^{6}\right)$ & .965085 & $\operatorname{Pr}\left(A \mid \pi_{A}^{1}, \pi_{B}^{2}, \pi_{A}^{3}, \pi_{B}^{4}, \pi_{B}^{5}, \sigma_{A}^{6}\right)$ & .577165 \\
\hline $\operatorname{Pr}\left(A \mid \pi_{A}^{1}, \pi_{A}^{2}, \pi_{A}^{3}, \pi_{B}^{4}, \pi_{A}^{5}, \sigma_{B}^{6}\right)$ & .948727 & $\operatorname{Pr}\left(A \mid \pi_{A}^{1}, \pi_{B}^{2}, \pi_{A}^{3}, \pi_{B}^{4}, \pi_{B}^{5}, \sigma_{B}^{6}\right)$ & 477467 \\
\hline $\operatorname{Pr}\left(A \mid \pi_{A}^{1}, \pi_{A}^{2}, \pi_{B}^{3}, \pi_{A}^{4}, \pi_{A}^{5}, \sigma_{A}^{6}\right)$ & .945976 & $\operatorname{Pr}\left(A \mid \pi_{A}^{1}, \pi_{B}^{2}, \pi_{B}^{3}, \pi_{A}^{4}, \pi_{B}^{5}, \sigma_{A}^{6}\right)$ & .463721 \\
\hline $\operatorname{Pr}\left(A \mid \pi_{A}^{1}, \pi_{A}^{2}, \pi_{B}^{3}, \pi_{A}^{4}, \pi_{A}^{5}, \sigma_{B}^{6}\right)$ & .921394 & $\operatorname{Pr}\left(A \mid \pi_{A}^{1}, \pi_{B}^{2}, \pi_{B}^{3}, \pi_{A}^{4}, \pi_{B}^{5}, \sigma_{B}^{6}\right)$ & \\
\hline $\operatorname{Pr}\left(A \mid \pi_{A}^{1}, \pi_{B}^{2}, \pi_{A}^{3}, \pi_{A}^{4}, \pi_{A}^{5}, \sigma_{A}^{6}\right)$ & .913738 & $\operatorname{Pr}\left(A \mid \pi_{A}^{1}, \pi_{B}^{2}, \pi_{B}^{3}, \pi_{B}^{4}, \pi_{A}^{5}, \sigma_{A}^{6}\right)$ & 656 \\
\hline $\mathrm{r}\left(A \mid \pi_{A}^{1}, \pi_{B}^{2}, \pi_{A}^{3}, \pi_{A}^{4}, \pi_{A}^{5}, \sigma_{B}^{6}\right)$ & .876404 & $\operatorname{Pr}\left(A \mid \pi_{A}^{1}, \pi_{B}^{2}, \pi_{B}^{3}, \pi_{B}^{4}, \pi_{A}^{5}, \sigma_{B}^{6}\right)$ & \\
\hline $\operatorname{Pr}\left(A \mid \pi_{B}^{1}, \pi_{A}^{2}, \pi_{A}^{3}, \pi_{A}^{4}, \pi_{A}^{5}, \sigma_{A}^{6}\right)$ & .856287 & $\operatorname{Pr}\left(A \mid \pi_{B}^{1}, \pi_{A}^{2}, \pi_{A}^{3}, \pi_{B}^{4}, \pi_{B}^{5}, \sigma_{A}^{6}\right)$ & .434 \\
\hline $\operatorname{Pr}\left(A \mid \pi_{B}^{1}, \pi_{A}^{2}, \pi_{A}^{3}, \pi_{A}^{4}, \pi_{A}^{5}, \sigma_{B}^{6}\right)$ & .799544 & $\operatorname{Pr}\left(A \mid \pi_{B}^{1}, \pi_{A}^{2}, \pi_{A}^{3}, \pi_{B}^{4}, \pi_{B}^{5}, \sigma_{B}^{6}\right)$ & \\
\hline $\operatorname{Pr}\left(A \mid \pi_{A}^{1}, \pi_{A}^{2}, \pi_{A}^{3}, \pi_{B}^{4}, \pi_{B}^{5}, \sigma_{A}^{6}\right)$ & .924727 & $\operatorname{Pr}\left(A \mid \pi_{B}^{1}, \pi_{A}^{2}, \pi_{B}^{3}, \pi_{A}^{4}, \pi_{B}^{5}, \sigma_{A}^{6}\right)$ & 327231 \\
\hline $\operatorname{Pr}\left(A \mid \pi_{A}^{1}, \pi_{A}^{2}, \pi_{A}^{3}, \pi_{B}^{4}, \pi_{B}^{5}, \sigma_{B}^{6}\right)$ & .891585 & $\operatorname{Pr}\left(A \mid \pi_{B}^{1}, \pi_{A}^{2}, \pi_{B}^{3}, \pi_{A}^{4}, \pi_{B}^{5}, \sigma_{B}^{6}\right)$ & 26 \\
\hline $\operatorname{Pr}\left(A \mid \pi_{A}^{1}, \pi_{A}^{2}, \pi_{B}^{3}, \pi_{B}^{4}, \pi_{A}^{5}, \sigma_{A}^{6}\right)$ & .835443 & $\operatorname{Pr}\left(A \mid \pi_{B}^{1}, \pi_{A}^{2}, \pi_{B}^{3}, \pi_{B}^{4}, \pi_{A}^{5}, \sigma_{A}^{6}\right)$ & .24 \\
\hline $\operatorname{Pr}\left(A \mid \pi_{A}^{1}, \pi_{A}^{2}, \pi_{B}^{3}, \pi_{B}^{4}, \pi_{A}^{5}, \sigma_{B}^{6}\right)$ & .772655 & $\operatorname{Pr}\left(A \mid \pi_{B}^{1}, \pi_{A}^{2}, \pi_{B}^{3}, \pi_{B}^{4}, \pi_{A}^{5}, \sigma_{B}^{6}\right)$ & .17 \\
\hline $\operatorname{Pr}\left(A \mid \pi_{A}^{1}, \pi_{B}^{2}, \pi_{B}^{3}, \pi_{A}^{4}, \pi_{A}^{5}, \sigma_{A}^{6}\right)$ & .660508 & $\operatorname{Pr}\left(A \mid \pi_{B}^{1}, \pi_{B}^{2}, \pi_{A}^{3}, \pi_{A}^{4}, \pi_{B}^{5}, \sigma_{A}^{6}\right)$ & .227 \\
\hline $\operatorname{Pr}\left(A \mid \pi_{A}^{1}, \pi_{B}^{2}, \pi_{B}^{3}, \pi_{A}^{4}, \pi_{A}^{5}, \sigma_{B}^{6}\right)$ & .565673 & $\operatorname{Pr}\left(A \mid \pi_{B}^{1}, \pi_{B}^{2}, \pi_{A}^{3}, \pi_{A}^{4}, \pi_{B}^{5}, \sigma_{B}^{6}\right)$ & 16 \\
\hline $\operatorname{Pr}\left(A \mid \pi_{B}^{1}, \pi_{B}^{2}, \pi_{A}^{3}, \pi_{A}^{4}, \pi_{A}^{5}, \sigma_{A}^{6}\right)$ & .398329 & $\operatorname{Pr}\left(A \mid \pi_{B}^{1}, \pi_{B}^{2}, \pi_{A}^{3}, \pi_{B}^{4}, \pi_{A}^{5}, \sigma_{A}^{6}\right)$ & .16104 \\
\hline $\operatorname{Pr}\left(A \mid \pi_{B}^{1}, \pi_{B}^{2}, \pi_{A}^{3}, \pi_{A}^{4}, \pi_{A}^{5}, \sigma_{B}^{6}\right)$ & .307087 & $\operatorname{Pr}\left(A \mid \pi_{B}^{1}, \pi_{B}^{2}, \pi_{A}^{3}, \pi_{B}^{4}, \pi_{A}^{5}, \sigma_{B}^{6}\right)$ & 113 \\
\hline $\operatorname{Pr}\left(A \mid \pi_{A}^{1}, \pi_{A}^{2}, \pi_{B}^{3}, \pi_{A}^{4}, \pi_{B}^{5}, \sigma_{A}^{6}\right)$ & .886135 & $\operatorname{Pr}\left(A \mid \pi_{B}^{1}, \pi_{B}^{2}, \pi_{B}^{3}, \pi_{A}^{4}, \pi_{A}^{5}, \sigma_{A}^{6}\right)$ & .108 \\
\hline $\mathrm{r}\left(A \mid \pi_{A}^{1}, \pi_{A}^{2}, \pi_{B}^{3}, \pi_{A}^{4}, \pi_{B}^{5}, \sigma_{B}^{6}\right)$ & .83896 & $\operatorname{Pr}\left(A \mid \pi_{B}^{1}, \pi_{B}^{2}, \pi_{B}^{3}, \pi_{A}^{4}, \pi_{A}^{5}, \sigma_{B}^{6}\right)$ & .075 \\
\hline $\operatorname{Pr}\left(A \mid \pi_{A}^{1}, \pi_{B}^{2}, \pi_{A}^{3}, \pi_{B}^{4}, \pi_{A}^{5}, \sigma_{A}^{6}\right)$ & .754374 & $\operatorname{Pr}\left(A \mid \pi_{A}^{1}, \pi_{B}^{2}, \pi_{B}^{3}, \pi_{B}^{4}, \pi_{B}^{5}, \sigma_{A}^{6}\right)$ & 456 \\
\hline $\operatorname{Pr}\left(A \mid \pi_{A}^{1}, \pi_{B}^{2}, \pi_{A}^{3}, \pi_{B}^{4}, \pi_{A}^{5}, \sigma_{B}^{6}\right)$ & .672769 & $\operatorname{Pr}\left(A \mid \pi_{A}^{1}, \pi_{B}^{2}, \pi_{B}^{3}, \pi_{B}^{4}, \pi_{B}^{5}, \sigma_{B}^{6}\right)$ & .143 \\
\hline $\operatorname{Pr}\left(A \mid \pi_{A}^{1}, \pi_{B}^{2}, \pi_{A}^{3}, \pi_{A}^{4}, \pi_{B}^{5}, \sigma_{A}^{6}\right)$ & .824802 & $\operatorname{Pr}\left(A \mid \pi_{B}^{1}, \pi_{A}^{2}, \pi_{B}^{3}, \pi_{B}^{4}, \pi_{B}^{5}, \sigma_{A}^{6}\right)$ & 3596 \\
\hline $\operatorname{Pr}\left(A \mid \pi_{A}^{1}, \pi_{B}^{2}, \pi_{A}^{3}, \pi_{A}^{4}, \pi_{B}^{5}, \sigma_{B}^{6}\right)$ & .759124 & $\operatorname{Pr}\left(A \mid \pi_{B}^{1}, \pi_{A}^{2}, \pi_{B}^{3}, \pi_{B}^{4}, \pi_{B}^{5}, \sigma_{B}^{6}\right)$ & .086262 \\
\hline $\operatorname{Pr}\left(A \mid \pi_{B}^{1}, \pi_{A}^{2}, \pi_{A}^{3}, \pi_{A}^{4}, \pi_{B}^{5}, \sigma_{A}^{6}\right)$ & .7258 & $\operatorname{Pr}\left(A \mid \pi_{B}^{1}, \pi_{B}^{2}, \pi_{A}^{3}, \pi_{B}^{4}, \pi_{B}^{5}, \sigma_{A}^{6}\right)$ & $.07 \%$ \\
\hline $\operatorname{Pr}\left(A \mid \pi_{B}^{1}, \pi_{A}^{2}, \pi_{A}^{3}, \pi_{A}^{4}, \pi_{B}^{5}, \sigma_{B}^{6}\right)$ & .639344 & $\operatorname{Pr}\left(A \mid \pi_{B}^{1}, \pi_{B}^{2}, \pi_{A}^{3}, \pi_{B}^{4}, \pi_{B}^{5}, \sigma_{B}^{6}\right)$ & .054024 \\
\hline
\end{tabular}

\begin{tabular}{|c|c|}
\hline $\operatorname{Pr}\left(A \mid \pi_{B}^{1}, \pi_{B}^{2}, \pi_{B}^{3}, \pi_{A}^{4}, \pi_{B}^{5}, \sigma_{A}^{6}\right)$ & .051273 \\
\hline $\operatorname{Pr}\left(A \mid \pi_{B}^{1}, \pi_{B}^{2}, \pi_{B}^{3}, \pi_{A}^{4}, \pi_{B}^{5}, \sigma_{B}^{6}\right)$ & .034915 \\
\hline $\operatorname{Pr}\left(A \mid \pi_{B}^{1}, \pi_{B}^{2}, \pi_{B}^{3}, \pi_{B}^{4}, \pi_{A}^{5}, \sigma_{A}^{6}\right)$ & .034056 \\
\hline $\operatorname{Pr}\left(A \mid \pi_{B}^{1}, \pi_{B}^{2}, \pi_{B}^{3}, \pi_{B}^{4}, \pi_{A}^{5}, \sigma_{B}^{6}\right)$ & .023057 \\
\hline $\operatorname{Pr}\left(A \mid \pi_{B}^{1}, \pi_{B}^{2}, \pi_{B}^{3}, \pi_{B}^{4}, \pi_{B}^{5}, \sigma_{A}^{6}\right)$ & .015428 \\
\hline $\operatorname{Pr}\left(A \mid \pi_{B}^{1}, \pi_{B}^{2}, \pi_{B}^{3}, \pi_{B}^{4}, \pi_{B}^{5}, \sigma_{B}^{6}\right)$ & .010381 \\
\hline
\end{tabular}

